\title{
Nickel complexes involved in the isomerization of 2-Methyl-3-Butenenitrile.
}

\author{
Alberto Acosta-Ramírez ${ }^{\dagger}$, Areli Flores-Gaspar ${ }^{\dagger}$, Miguel Muñoz-Hernández ${ }^{\S}$, Alma Arévalo ${ }^{\dagger}$, \\ William D. Jones ${ }^{\star}$ and Juventino J. García ${ }^{\dagger, *}$ \\ † Facultad de Química, Universidad Nacional Autónoma de México, México City, MéxicoD. F. \\ 04510. México \\ ${ }^{\S}$ Centro de Investigaciones Químicas, Universidad Autónoma del Estado de Morelos, \\ Cuernavaca, Morelos, 62210. México. \\ * Department of Chemistry. University of Rochester, Rochester, New york, 14627, USA.
}

Figure S-1. GC-MS Determinations.

Figure S-2. ORTEP drawing of complex 6' showing 50\% ellipsoids.

Table S-1. Crystallographic data for complex 6'.

Table S-2. Positional parameters and U(eq) for complex 6'.

Table S-3. Intramolecular distances $(\AA)$ and bond angles $\left(^{\circ}\right)$ for complex $\mathbf{6}^{\prime}$.

Table S-4. U(aniso) values for complex 6'.

Table S-5. Hydrogen atom coordinates for complex 6'.

Figure S-3. ORTEP drawing of complex 7' showing 50\% ellipsoids.

Table S-6. Crystallographic data for complex 7'.

Table S-7. Positional parameters and U(eq) for complex 7'.

Table S-8. Intramolecular distances $(\AA)$ and bond angles $\left(^{\circ}\right)$ for complex 7'.

Table S-9. U(aniso) values for complex 7'.

Table S-10. Hydrogen atom coordinates for complex 7'.

Figure S-4. ORTEP drawing of complex 10 showing 50\% ellipsoids.

Table S-11. Crystallographic data for complex 10.

Table S-12. Positional parameters and U(eq) for complex 10.

Table S-13. Intramolecular distances $(\AA)$ and bond angles $\left(^{\circ}\right)$ for complex 10. 
Table S-14. U(aniso) values for complex 10.

Table S-15. Hydrogen atom coordinates for complex 10.

Figure S-5. NMR spectra for 1'.

Figure S-6. NMR spectra for 2.

Figure S-7. NMR spectra for 3 and 4.

Figure S-8. NMR spectra for 5.

Figure S-9. NMR spectra for 6.

Figure S-10. NMR spectra for 6'.

Figure S-11. NMR spectra for 7.

Figure S-12. NMR spectra for 7' 

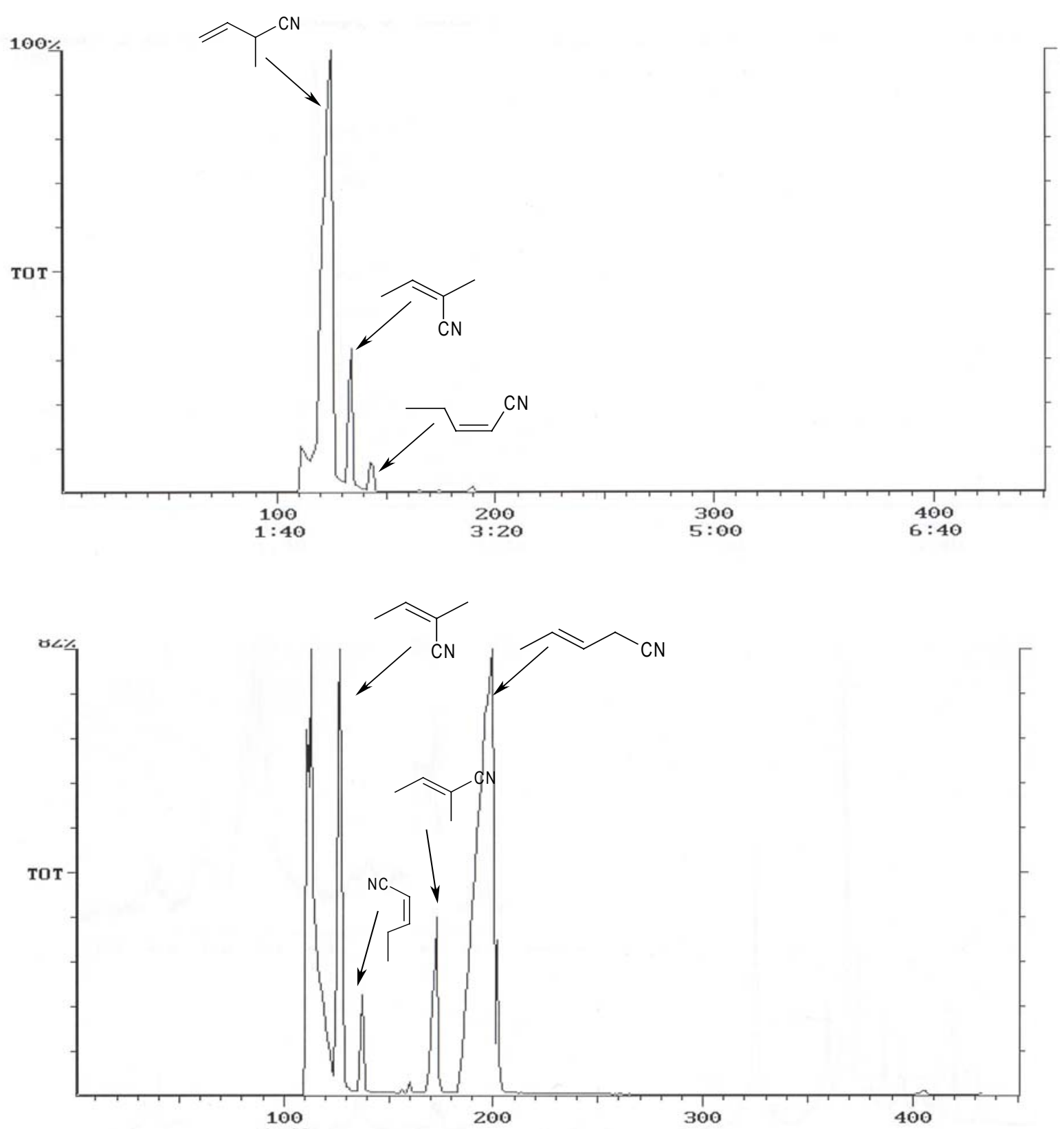

Figure S-1. GC-MS Determinatıons: top: analysis of reactant $2 \mathrm{M} 3 \mathrm{BN}$; bottom: analysis of catalytic reaction using catalyst as described in Table 4.

The samples from catalytic experiments were analyzed by GC-MS on a 30m DB-5MS capillary column, ID $0.32 \mathrm{~mm}$, film 0.25 microns, $40 \mathrm{~mL} / \mathrm{min}$, using an isothermal $\mathrm{GC}$ method at $35^{\circ} \mathrm{C}$ during 8 minutes, followed by a heating at $11.8^{\circ} \mathrm{C} / \mathrm{min}$ during 6.35 minutes, to reach $110{ }^{\circ} \mathrm{C}$, being stable at that temperature for 3 more minutes. All the nitriles present in the samples are detected during the isothermal part of the method. 


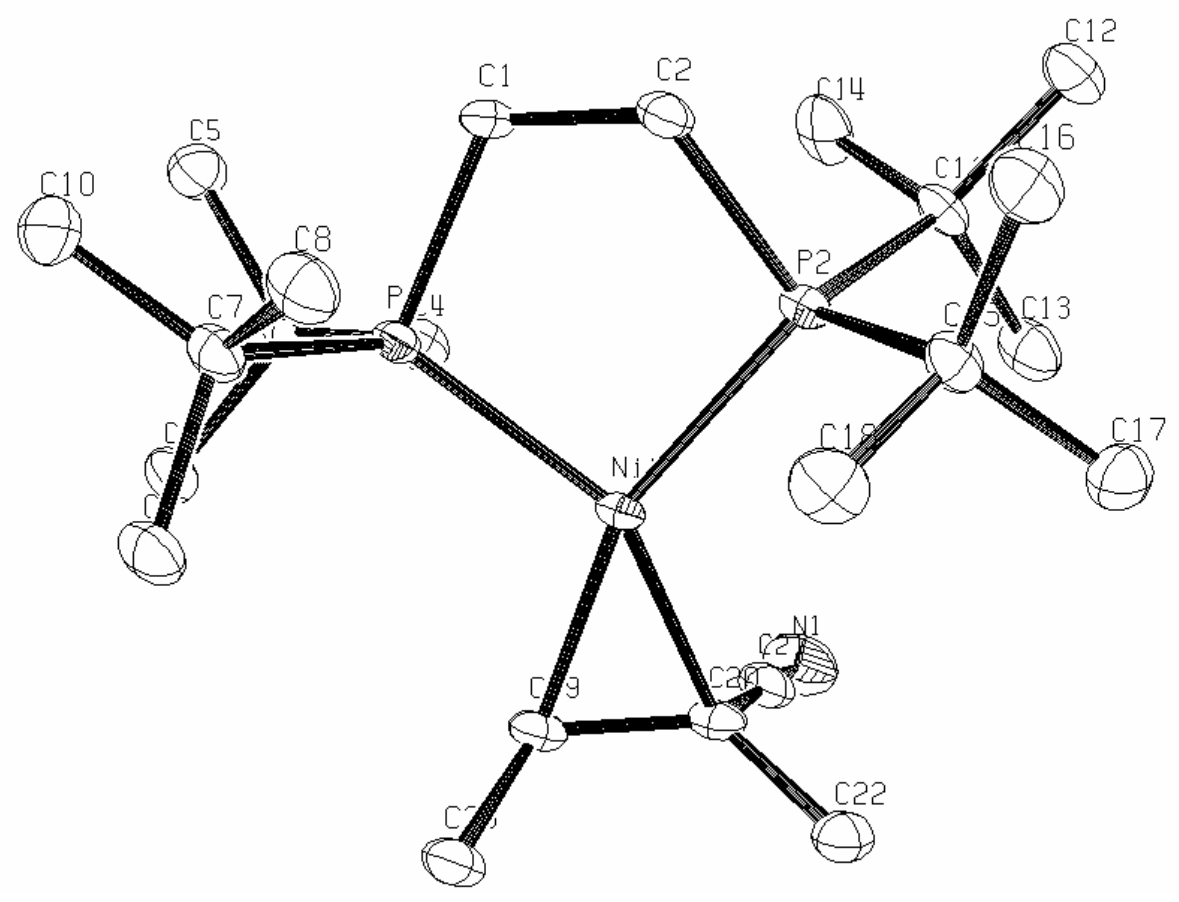

Figure S-2. ORTEP drawing of complex 6' showing 50\% ellipsoids. 
Table S-1. Crystallographic data for complex 6'

Identification code

Empirical formula

Formula weight

Temperature

Wavelength

Crystal system

Space group

Unit cell dimensions

Volume

Z

Density (calculated)

Absorption coefficient

$\mathrm{F}(000)$

Crystal size

Theta range for data collection

Index ranges

Reflections collected

Independent reflections

Completeness to theta $=24.99^{\circ}$

Absorption correction

Refinement method

Data / restraints / parameters

Goodness-of-fit on $\mathrm{F}^{2}$

Final $\mathrm{R}$ indices [I $>2 \operatorname{sigma}(\mathrm{I})]$

$\mathrm{R}$ indices (all data)

Largest diff. peak and hole juv556m

$\mathrm{C}_{23} \mathrm{H}_{47} \mathrm{NNiP}_{2}$

458.27

100(2) K

$0.71073 \AA$

Monoclinic

$\mathrm{P} 2(1) / \mathrm{n}$

$a=11.1322(15) \AA$

$\alpha=90^{\circ}$.

$\mathrm{b}=14.1435(19) \AA$

$\beta=102.732(2)^{\circ}$.

$\mathrm{c}=16.532(2) \AA$

$\gamma=90^{\circ}$.
4

$1.199 \mathrm{Mg} / \mathrm{m}^{3}$

$0.899 \mathrm{~mm}^{-1}$

1000

$0.31 \times 0.23 \times 0.17 \mathrm{~mm}^{3}$

1.92 to $24.99^{\circ}$.

$-5<=\mathrm{h}<=12,-11<=\mathrm{k}<=16,-19<=\mathrm{l}<=19$

6320

$4050[\mathrm{R}(\mathrm{int})=0.0198]$

$90.6 \%$

None

Full-matrix least-squares on $\mathrm{F}^{2}$

4050 / 0 / 258

0.679

$\mathrm{R} 1=0.0311, \mathrm{wR} 2=0.0856$

$\mathrm{R} 1=0.0349, \mathrm{wR} 2=0.0896$

0.563 and -0.252 e. $\AA^{-3}$ 
Table S-2. Positional parameters and U(eq) for complex 6'

\begin{tabular}{|c|c|c|c|c|}
\hline & $\mathrm{x}$ & $\mathrm{y}$ & $\mathrm{z}$ & $\mathrm{U}(\mathrm{eq})$ \\
\hline $\mathrm{Ni}(1)$ & $3264(1)$ & 1723(1) & $849(1)$ & $13(1)$ \\
\hline $\mathrm{P}(1)$ & 4909(1) & $2448(1)$ & $1547(1)$ & $14(1)$ \\
\hline $\mathrm{P}(2)$ & 2049(1) & $2454(1)$ & $1536(1)$ & $14(1)$ \\
\hline $\mathrm{C}(1)$ & 4413(2) & $3006(1)$ & $2438(1)$ & $18(1)$ \\
\hline$C(19)$ & $3768(2)$ & $908(1)$ & $24(1)$ & $18(1)$ \\
\hline$C(2)$ & $3055(2)$ & $3341(1)$ & $2195(1)$ & $18(1)$ \\
\hline $\mathrm{C}(9)$ & 5929(2) & $3193(2)$ & $259(1)$ & $25(1)$ \\
\hline $\mathrm{C}(11)$ & $1324(2)$ & $1787(1)$ & 2303(1) & 19(1) \\
\hline$C(15)$ & $827(2)$ & $3222(1)$ & $851(1)$ & 19(1) \\
\hline$C(6)$ & $6881(2)$ & 1219(1) & $1458(1)$ & $21(1)$ \\
\hline$C(3)$ & $6114(2)$ & $1556(1)$ & $2068(1)$ & $18(1)$ \\
\hline $\mathrm{C}(22)$ & $1421(2)$ & $895(1)$ & $-635(1)$ & $22(1)$ \\
\hline$C(17)$ & $-311(2)$ & $2637(2)$ & $451(1)$ & $27(1)$ \\
\hline $\mathrm{C}(10)$ & $6952(2)$ & $3769(2)$ & $1679(1)$ & $27(1)$ \\
\hline $\mathrm{C}(23)$ & $4042(2)$ & $1340(2)$ & $-751(1)$ & $24(1)$ \\
\hline$C(4)$ & $5393(2)$ & $704(1)$ & $2300(1)$ & $21(1)$ \\
\hline $\mathrm{C}(20)$ & $2523(2)$ & $690(1)$ & $78(1)$ & $18(1)$ \\
\hline $\mathrm{C}(18)$ & $1423(2)$ & $3590(2)$ & $152(1)$ & $28(1)$ \\
\hline $\mathrm{C}(21)$ & $2390(2)$ & $-168(1)$ & $529(1)$ & $21(1)$ \\
\hline$C(5)$ & $6983(2)$ & 1893(2) & $2875(1)$ & $24(1)$ \\
\hline$C(7)$ & $5726(2)$ & $3453(1)$ & $1120(1)$ & $19(1)$ \\
\hline$C(14)$ & $2387(2)$ & $1439(2)$ & 2999(1) & $28(1)$ \\
\hline $\mathrm{C}(8)$ & $4857(2)$ & $4318(1)$ & $1005(1)$ & $26(1)$ \\
\hline$C(12)$ & $454(2)$ & $2372(2)$ & $2705(1)$ & $27(1)$ \\
\hline$C(13)$ & $644(2)$ & $912(2)$ & $1872(1)$ & $27(1)$ \\
\hline$C(16)$ & $414(2)$ & $4084(1)$ & $1288(1)$ & $28(1)$ \\
\hline $\mathrm{N}(1)$ & $2298(2)$ & $-865(1)$ & $869(1)$ & $33(1)$ \\
\hline
\end{tabular}


Table S-3. Intramolecular distances $(\AA)$ and bond angles $\left(^{\circ}\right)$ for complex $\mathbf{6}^{\prime}$.

\begin{tabular}{|c|c|c|c|}
\hline $\mathrm{Ni}(1)-\mathrm{C}(19)$ & $1.9590(19)$ & $\mathrm{C}(11)-\mathrm{C}(12)$ & $1.532(3)$ \\
\hline $\mathrm{Ni}(1)-\mathrm{C}(20)$ & $1.9936(18)$ & $\mathrm{C}(11)-\mathrm{C}(14)$ & $1.539(3)$ \\
\hline $\mathrm{Ni}(1)-\mathrm{P}(1)$ & $2.1930(6)$ & $\mathrm{C}(15)-\mathrm{C}(18)$ & $1.545(3)$ \\
\hline $\mathrm{Ni}(1)-\mathrm{P}(2)$ & $2.2072(6)$ & $C(15)-C(16)$ & $1.539(3)$ \\
\hline $\mathrm{P}(1)-\mathrm{C}(1)$ & $1.859(2)$ & $\mathrm{C}(15)-\mathrm{C}(17)$ & $1.535(3)$ \\
\hline $\mathrm{P}(1)-\mathrm{C}(7)$ & $1.905(2)$ & $C(6)-C(3)$ & $1.534(3)$ \\
\hline $\mathrm{P}(1)-\mathrm{C}(3)$ & $1.902(2)$ & $C(3)-C(4)$ & $1.543(3)$ \\
\hline $\mathrm{P}(2)-\mathrm{C}(2)$ & $1.8646(19)$ & $C(3)-C(5)$ & $1.540(3)$ \\
\hline $\mathrm{P}(2)-\mathrm{C}(11)$ & $1.897(2)$ & $C(22)-C(20)$ & $1.531(3)$ \\
\hline $\mathrm{P}(2)-\mathrm{C}(15)$ & $1.9058(19)$ & $\mathrm{C}(10)-\mathrm{C}(7)$ & $1.536(3)$ \\
\hline$C(1)-C(2)$ & $1.550(3)$ & $C(20)-C(21)$ & $1.448(3)$ \\
\hline$C(19)-C(20)$ & $1.442(3)$ & $\mathrm{C}(21)-\mathrm{N}(1)$ & $1.150(3)$ \\
\hline$C(19)-C(23)$ & $1.510(3)$ & $C(7)-C(8)$ & $1.545(3)$ \\
\hline$C(9)-C(7)$ & $1.534(3)$ & & \\
\hline$C(11)-C(13)$ & $1.541(3)$ & & \\
\hline C(19)-Ni(1)-C(20) & $42.77(8)$ & $\mathrm{C}(20)-\mathrm{C}(19)-\mathrm{C}(23)$ & $121.05(17)$ \\
\hline $\mathrm{C}(19)-\mathrm{Ni}(1)-\mathrm{P}(1)$ & 107.91(6) & $\mathrm{C}(20)-\mathrm{C}(19)-\mathrm{Ni}(1)$ & $69.89(11)$ \\
\hline $\mathrm{C}(20)-\mathrm{Ni}(1)-\mathrm{P}(1)$ & $149.23(6)$ & $\mathrm{C}(23)-\mathrm{C}(19)-\mathrm{Ni}(1)$ & $119.70(14)$ \\
\hline $\mathrm{C}(19)-\mathrm{Ni}(1)-\mathrm{P}(2)$ & $159.44(6)$ & $\mathrm{C}(1)-\mathrm{C}(2)-\mathrm{P}(2)$ & $111.86(13)$ \\
\hline $\mathrm{C}(20)-\mathrm{Ni}(1)-\mathrm{P}(2)$ & $117.29(6)$ & $\mathrm{C}(13)-\mathrm{C}(11)-\mathrm{C}(12)$ & $109.95(17)$ \\
\hline $\mathrm{P}(1)-\mathrm{Ni}(1)-\mathrm{P}(2)$ & $92.58(2)$ & $\mathrm{C}(13)-\mathrm{C}(11)-\mathrm{C}(14)$ & $107.86(17)$ \\
\hline $\mathrm{C}(1)-\mathrm{P}(1)-\mathrm{C}(7)$ & $103.05(9)$ & $\mathrm{C}(12)-\mathrm{C}(11)-\mathrm{C}(14)$ & $107.70(17)$ \\
\hline $\mathrm{C}(1)-\mathrm{P}(1)-\mathrm{C}(3)$ & $103.08(9)$ & $\mathrm{C}(13)-\mathrm{C}(11)-\mathrm{P}(2)$ & $109.20(14)$ \\
\hline $\mathrm{C}(7)-\mathrm{P}(1)-\mathrm{C}(3)$ & $108.41(9)$ & $\mathrm{C}(12)-\mathrm{C}(11)-\mathrm{P}(2)$ & $115.10(14)$ \\
\hline $\mathrm{C}(1)-\mathrm{P}(1)-\mathrm{Ni}(1)$ & $104.87(6)$ & $\mathrm{C}(14)-\mathrm{C}(11)-\mathrm{P}(2)$ & $106.75(15)$ \\
\hline $\mathrm{C}(7)-\mathrm{P}(1)-\mathrm{Ni}(1)$ & $124.41(6)$ & $\mathrm{C}(18)-\mathrm{C}(15)-\mathrm{C}(16)$ & $107.78(17)$ \\
\hline $\mathrm{C}(3)-\mathrm{P}(1)-\mathrm{Ni}(1)$ & $110.61(6)$ & $\mathrm{C}(18)-\mathrm{C}(15)-\mathrm{C}(17)$ & 108.01(17) \\
\hline $\mathrm{C}(2)-\mathrm{P}(2)-\mathrm{C}(11)$ & $103.55(9)$ & $\mathrm{C}(16)-\mathrm{C}(15)-\mathrm{C}(17)$ & $108.98(17)$ \\
\hline $\mathrm{C}(2)-\mathrm{P}(2)-\mathrm{C}(15)$ & $102.83(9)$ & $\mathrm{C}(18)-\mathrm{C}(15)-\mathrm{P}(2)$ & $105.61(14)$ \\
\hline $\mathrm{C}(11)-\mathrm{P}(2)-\mathrm{C}(15)$ & $109.12(9)$ & $\mathrm{C}(16)-\mathrm{C}(15)-\mathrm{P}(2)$ & $114.86(14)$ \\
\hline $\mathrm{C}(2)-\mathrm{P}(2)-\mathrm{Ni}(1)$ & $104.96(7)$ & $\mathrm{C}(17)-\mathrm{C}(15)-\mathrm{P}(2)$ & $111.28(13)$ \\
\hline $\mathrm{C}(11)-\mathrm{P}(2)-\mathrm{Ni}(1)$ & $120.70(6)$ & $\mathrm{C}(4)-\mathrm{C}(3)-\mathrm{C}(6)$ & $108.40(16)$ \\
\hline $\mathrm{C}(15)-\mathrm{P}(2)-\mathrm{Ni}(1)$ & $113.49(7)$ & $\mathrm{C}(4)-\mathrm{C}(3)-\mathrm{C}(5)$ & $106.76(16)$ \\
\hline $\mathrm{C}(2)-\mathrm{C}(1)-\mathrm{P}(1)$ & $111.53(12)$ & $\mathrm{C}(6)-\mathrm{C}(3)-\mathrm{C}(5)$ & $109.33(17)$ \\
\hline
\end{tabular}




$\begin{array}{lc}\mathrm{C}(4)-\mathrm{C}(3)-\mathrm{P}(1) & 106.06(13) \\ \mathrm{C}(6)-\mathrm{C}(3)-\mathrm{P}(1) & 110.36(13) \\ \mathrm{C}(5)-\mathrm{C}(3)-\mathrm{P}(1) & 115.61(14) \\ \mathrm{C}(21)-\mathrm{C}(20)-\mathrm{C}(19) & 114.86(17) \\ \mathrm{C}(21)-\mathrm{C}(20)-\mathrm{C}(22) & 113.55(17) \\ \mathrm{C}(19)-\mathrm{C}(20)-\mathrm{C}(22) & 122.02(17) \\ \mathrm{C}(21)-\mathrm{C}(20)-\mathrm{Ni}(1) & 111.08(13) \\ \mathrm{C}(19)-\mathrm{C}(20)-\mathrm{Ni}(1) & 67.33(10) \\ \mathrm{C}(22)-\mathrm{C}(20)-\mathrm{Ni}(1) & 120.11(13) \\ \mathrm{N}(1)-\mathrm{C}(21)-\mathrm{C}(20) & 177.9(2) \\ \mathrm{C}(9)-\mathrm{C}(7)-\mathrm{C}(8) & 106.97(17) \\ \mathrm{C}(9)-\mathrm{C}(7)-\mathrm{C}(10) & 109.51(18) \\ \mathrm{C}(8)-\mathrm{C}(7)-\mathrm{C}(10) & 107.03(16) \\ \mathrm{C}(9)-\mathrm{C}(7)-\mathrm{P}(1) & 110.16(13) \\ \mathrm{C}(8)-\mathrm{C}(7)-\mathrm{P}(1) & 107.46(14) \\ \mathrm{C}(10)-\mathrm{C}(7)-\mathrm{P}(1) & 115.32(14)\end{array}$


Table S-4. U(aniso) values for complex 6'

\begin{tabular}{|c|c|c|c|c|c|c|}
\hline & $\mathrm{U}^{11}$ & $\mathrm{U}^{22}$ & $\mathrm{U}^{33}$ & $\mathrm{U}^{23}$ & $\mathrm{U}^{13}$ & $\mathrm{U}^{12}$ \\
\hline $\mathrm{Ni}(1)$ & $17(1)$ & $15(1)$ & $10(1)$ & $-2(1)$ & $5(1)$ & $1(1)$ \\
\hline $\mathrm{P}(1)$ & $16(1)$ & $16(1)$ & $12(1)$ & $-1(1)$ & $6(1)$ & $0(1)$ \\
\hline $\mathrm{P}(2)$ & $17(1)$ & $15(1)$ & $13(1)$ & $-1(1)$ & $6(1)$ & $1(1)$ \\
\hline $\mathrm{C}(1)$ & $21(1)$ & $21(1)$ & $12(1)$ & $-6(1)$ & $5(1)$ & $-2(1)$ \\
\hline$C(19)$ & $23(1)$ & $19(1)$ & $14(1)$ & $-4(1)$ & $6(1)$ & $3(1)$ \\
\hline $\mathrm{C}(2)$ & $21(1)$ & $17(1)$ & $18(1)$ & $-4(1)$ & $9(1)$ & $0(1)$ \\
\hline $\mathrm{C}(9)$ & $31(1)$ & $25(1)$ & $23(1)$ & $1(1)$ & $14(1)$ & $-3(1)$ \\
\hline $\mathrm{C}(11)$ & $20(1)$ & $24(1)$ & $17(1)$ & $1(1)$ & $12(1)$ & $1(1)$ \\
\hline$C(15)$ & $19(1)$ & $19(1)$ & $20(1)$ & $2(1)$ & $6(1)$ & $6(1)$ \\
\hline$C(6)$ & $19(1)$ & $23(1)$ & $23(1)$ & $-1(1)$ & $8(1)$ & $4(1)$ \\
\hline $\mathrm{C}(3)$ & $18(1)$ & $21(1)$ & $16(1)$ & $0(1)$ & $4(1)$ & $1(1)$ \\
\hline $\mathrm{C}(22)$ & $25(1)$ & $22(1)$ & $19(1)$ & $-5(1)$ & $6(1)$ & $-1(1)$ \\
\hline$C(17)$ & $23(1)$ & $28(1)$ & $26(1)$ & $-3(1)$ & $1(1)$ & $3(1)$ \\
\hline$C(10)$ & $28(1)$ & $26(1)$ & $28(1)$ & $-1(1)$ & $9(1)$ & $-8(1)$ \\
\hline$C(23)$ & $29(1)$ & $28(1)$ & $17(1)$ & $-5(1)$ & $10(1)$ & $-2(1)$ \\
\hline $\mathrm{C}(4)$ & $22(1)$ & $21(1)$ & $20(1)$ & $2(1)$ & $6(1)$ & $4(1)$ \\
\hline$C(20)$ & $25(1)$ & $15(1)$ & $14(1)$ & $-5(1)$ & $6(1)$ & $0(1)$ \\
\hline $\mathrm{C}(18)$ & $32(1)$ & $28(1)$ & $23(1)$ & $8(1)$ & $6(1)$ & $6(1)$ \\
\hline $\mathrm{C}(21)$ & $25(1)$ & $22(1)$ & $19(1)$ & $-5(1)$ & $10(1)$ & $0(1)$ \\
\hline$C(5)$ & $22(1)$ & $30(1)$ & $20(1)$ & $0(1)$ & $3(1)$ & $3(1)$ \\
\hline$C(7)$ & $23(1)$ & $18(1)$ & $20(1)$ & $1(1)$ & $11(1)$ & $-2(1)$ \\
\hline $\mathrm{C}(14)$ & $26(1)$ & $38(1)$ & $23(1)$ & $12(1)$ & $12(1)$ & $1(1)$ \\
\hline $\mathrm{C}(8)$ & $32(1)$ & $21(1)$ & $27(1)$ & $3(1)$ & $13(1)$ & $-1(1)$ \\
\hline $\mathrm{C}(12)$ & $27(1)$ & $33(1)$ & $25(1)$ & $-2(1)$ & $15(1)$ & $0(1)$ \\
\hline$C(13)$ & $31(1)$ & $24(1)$ & $30(1)$ & 1(1) & $17(1)$ & $-4(1)$ \\
\hline$C(16)$ & $27(1)$ & $20(1)$ & $37(1)$ & $-3(1)$ & $7(1)$ & $9(1)$ \\
\hline $\mathrm{N}(1)$ & $47(1)$ & $27(1)$ & $30(1)$ & $3(1)$ & $15(1)$ & $-1(1)$ \\
\hline
\end{tabular}


Table S-5. Hydrogen atom coordinates for complex 6 '.

\begin{tabular}{|c|c|c|c|c|}
\hline & $\mathrm{x}$ & $\mathrm{y}$ & $\mathrm{z}$ & $\mathrm{U}(\mathrm{eq})$ \\
\hline $\mathrm{H}(1 \mathrm{~A})$ & 4504 & 2555 & 2889 & 21 \\
\hline $\mathrm{H}(1 \mathrm{~B})$ & 4940 & 3543 & 2632 & 21 \\
\hline H(19) & 4363 & 421 & 271 & 22 \\
\hline $\mathrm{H}(2 \mathrm{~A})$ & 3015 & 3934 & 1895 & 22 \\
\hline $\mathrm{H}(2 \mathrm{~B})$ & 2753 & 3453 & 2694 & 22 \\
\hline $\mathrm{H}(9 \mathrm{~A})$ & 6345 & 3702 & 51 & 37 \\
\hline $\mathrm{H}(9 \mathrm{~B})$ & 5148 & 3083 & -111 & 37 \\
\hline $\mathrm{H}(9 \mathrm{C})$ & 6422 & 2630 & 300 & 37 \\
\hline $\mathrm{H}(6 \mathrm{~A})$ & 7386 & 1729 & 1341 & 32 \\
\hline $\mathrm{H}(6 \mathrm{~B})$ & 6341 & 1015 & 953 & 32 \\
\hline $\mathrm{H}(6 \mathrm{C})$ & 7396 & 702 & 1698 & 32 \\
\hline $\mathrm{H}(22 \mathrm{~A})$ & 1448 & 485 & -1094 & 33 \\
\hline $\mathrm{H}(22 \mathrm{~B})$ & 1451 & 1542 & -806 & 33 \\
\hline $\mathrm{H}(22 \mathrm{C})$ & 671 & 787 & -453 & 33 \\
\hline $\mathrm{H}(17 \mathrm{~A})$ & -739 & 2448 & 869 & 40 \\
\hline $\mathrm{H}(17 \mathrm{~B})$ & -58 & 2086 & 193 & 40 \\
\hline $\mathrm{H}(17 \mathrm{C})$ & -848 & 3013 & 40 & 40 \\
\hline $\mathrm{H}(10 \mathrm{~A})$ & 7547 & 3270 & 1715 & 40 \\
\hline $\mathrm{H}(10 \mathrm{~B})$ & 6830 & 3911 & 2223 & 40 \\
\hline $\mathrm{H}(10 \mathrm{C})$ & 7246 & 4323 & 1447 & 40 \\
\hline $\mathrm{H}(23 \mathrm{~A})$ & 3770 & 918 & -1211 & 35 \\
\hline $\mathrm{H}(23 \mathrm{~B})$ & 4912 & 1442 & -673 & 35 \\
\hline $\mathrm{H}(23 \mathrm{C})$ & 3617 & 1932 & -863 & 35 \\
\hline $\mathrm{H}(4 \mathrm{~A})$ & 5956 & 202 & 2508 & 32 \\
\hline $\mathrm{H}(4 \mathrm{~B})$ & 4810 & 490 & 1817 & 32 \\
\hline $\mathrm{H}(4 \mathrm{C})$ & 4964 & 890 & 2718 & 32 \\
\hline $\mathrm{H}(18 \mathrm{~A})$ & 1793 & 3073 & -79 & 41 \\
\hline $\mathrm{H}(18 \mathrm{~B})$ & 2043 & 4049 & 374 & 41 \\
\hline $\mathrm{H}(18 \mathrm{C})$ & 804 & 3878 & -273 & 41 \\
\hline $\mathrm{H}(5 \mathrm{~A})$ & 7498 & 1378 & 3120 & 36 \\
\hline $\mathrm{H}(5 \mathrm{~B})$ & 6505 & 2111 & 3255 & 36 \\
\hline
\end{tabular}




\begin{tabular}{lrrrr}
$\mathrm{H}(5 \mathrm{C})$ & 7488 & 2401 & 2752 & 36 \\
$\mathrm{H}(14 \mathrm{~A})$ & 2775 & 1970 & 3313 & 42 \\
$\mathrm{H}(14 \mathrm{~B})$ & 2980 & 1110 & 2760 & 42 \\
$\mathrm{H}(14 \mathrm{C})$ & 2071 & 1018 & 3358 & 42 \\
$\mathrm{H}(8 \mathrm{~A})$ & 5228 & 4830 & 766 & 38 \\
$\mathrm{H}(8 \mathrm{~B})$ & 4718 & 4509 & 1534 & 38 \\
$\mathrm{H}(8 \mathrm{C})$ & 4087 & 4153 & 644 & 38 \\
$\mathrm{H}(12 \mathrm{~A})$ & 220 & 2008 & 3135 & 41 \\
$\mathrm{H}(12 \mathrm{~B})$ & -267 & 2536 & 2294 & 41 \\
$\mathrm{H}(12 \mathrm{C})$ & 866 & 2938 & 2938 & 41 \\
$\mathrm{H}(13 \mathrm{~A})$ & 290 & 565 & 2262 & 40 \\
$\mathrm{H}(13 \mathrm{~B})$ & 1215 & 516 & 1672 & 40 \\
$\mathrm{H}(13 \mathrm{C})$ & 2 & 1110 & 1415 & 40 \\
$\mathrm{H}(16 \mathrm{~A})$ & -165 & 4449 & 894 & 42 \\
$\mathrm{H}(16 \mathrm{~B})$ & 1117 & 4468 & 1519 & 42 \\
$\mathrm{H}(16 \mathrm{C})$ & 32 & 3874 & 1723 & 42 \\
& & & & \\
\hline
\end{tabular}




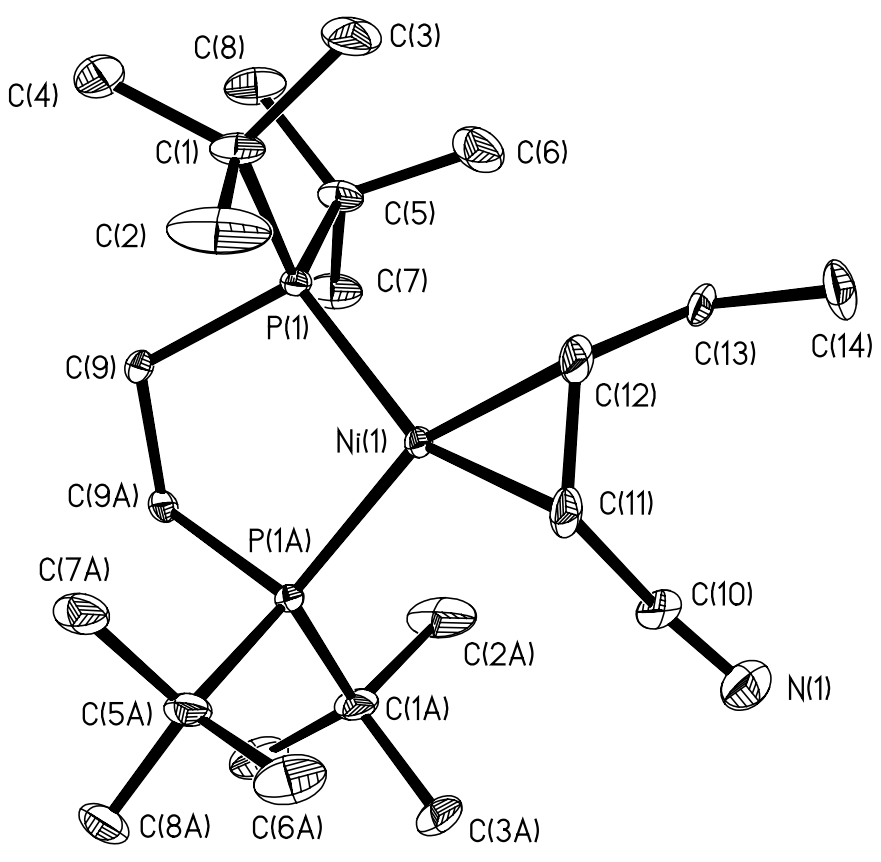

Fig. S-3. ORTEP drawing of complex $\mathbf{7}^{\prime}$ showing $50 \%$ ellipsoids. The nitrile ligand and nickel are disordered, and only one of two orientations is shown. Hydrogen atoms have been omitted.

Details of solution: The molecule crystallizes in space group $\mathrm{C} 2 / \mathrm{c}$ with $Z=4$. The nickel lies slightly off of the 2fold, which generates a second nickel (population 50\%) and a second orientation of the nitrile ligand (population $50 \%$ ). The dtbpe ligand was refined in a single orientation. Hydrogens were placed in ideal locations on the dtbpe ligand, but were omitted on the nitrile ligand.

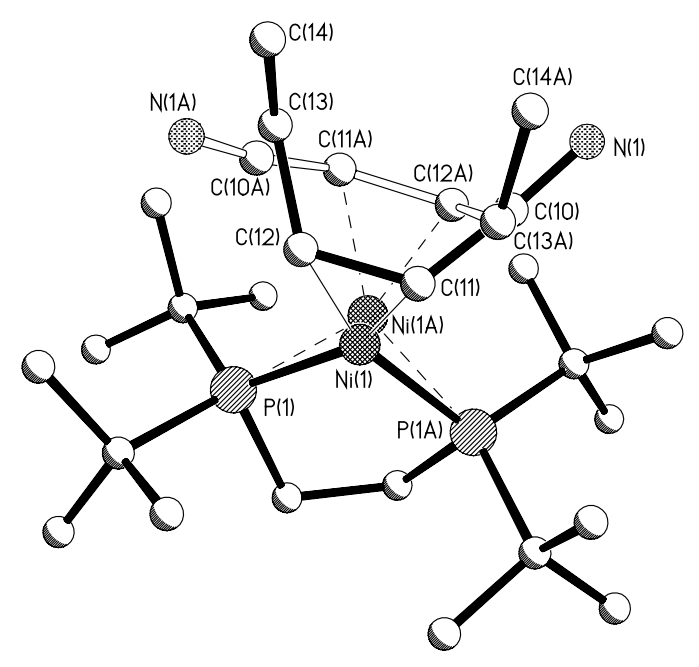


Table S-6. Crystallographic data for complex 7'.
Identification code
jaa44m
Empirical formula
C23 H46 N Ni P2
Formula weight
457.26
Temperature
$100(2) \mathrm{K}$
Wavelength
$0.71073 \mathrm{~A}$
Crystal system
Monoclinic
Space group
$\mathrm{C} 2 / \mathrm{c}$
Unit cell dimensions
$\mathrm{a}=18.886(4) \AA \quad$ alpha $=90 \mathrm{deg}$.
$\mathrm{b}=10.536(2) \AA$ beta $=91.843(4) \mathrm{deg}$.
$\mathrm{c}=12.667(3) \AA$ gamma $=90 \mathrm{deg}$.
Volume, Z
2519.2(10) $\AA^{3}, 4$
Density (calculated)
$1.206 \mathrm{Mg} / \mathrm{m}^{3}$
Absorption coefficient
$0.906 \mathrm{~mm}^{-1}$
$\mathrm{F}(000)$
996
Crystal size
$0.2 \times 0.14 \times 0.1 \mathrm{~mm}$
Theta range for data collection 2.16 to $28.27 \mathrm{deg}$.
Limiting indices
$-25<=\mathrm{h}<=24,-13<=\mathrm{k}<=13,-16<=\mathrm{l}<=16$
Reflections collected
11295
Independent reflections
$2993[\mathrm{R}(\mathrm{int})=0.0604]$
Absorption correction
None
Refinement method
Full-matrix least-squares on $\mathrm{F}^{\wedge} 2$
Data / restraints / parameters
$2993 / 0 / 154$
Goodness-of-fit on $\mathrm{F}^{\wedge} 2$
1.000
Final $R$ indices [I $>2 \operatorname{sigma}(\mathrm{I})]$
$\mathrm{R} 1=0.0562, \mathrm{wR} 2=0.1322$
$\mathrm{R}$ indices (all data)
$\mathrm{R} 1=0.0808, \mathrm{wR} 2=0.1430$
Largest diff. peak and hole
0.466 and -0.304 e. $\mathrm{A}^{-3}$ 
Table S-7. Positional parameters and U(eq) for complex 7'.

\begin{tabular}{lrrrl}
\hline & & & & \\
& $\mathrm{x}$ & $\mathrm{y}$ & $\mathrm{U}(\mathrm{eq})$ \\
& & & & \\
$\mathrm{Ni}(1)$ & $30(1)$ & $1169(1)$ & $2703(1)$ & $15(1)$ \\
$\mathrm{P}(1)$ & $823(1)$ & $2583(1)$ & $2315(1)$ & $17(1)$ \\
$\mathrm{C}(1)$ & $1577(2)$ & $2508(3)$ & $3324(3)$ & $31(1)$ \\
$\mathrm{C}(2)$ & $1239(2)$ & $2085(6)$ & $4350(3)$ & $70(2)$ \\
$\mathrm{C}(3)$ & $2109(2)$ & $1498(4)$ & $3026(3)$ & $45(1)$ \\
$\mathrm{C}(4)$ & $1967(2)$ & $3751(4)$ & $3527(3)$ & $46(1)$ \\
$\mathrm{C}(5)$ & $1192(2)$ & $2842(3)$ & $969(2)$ & $29(1)$ \\
$\mathrm{C}(6)$ & $1412(2)$ & $1563(4)$ & $507(3)$ & $50(1)$ \\
$\mathrm{C}(7)$ & $599(2)$ & $3373(4)$ & $251(3)$ & $39(1)$ \\
$\mathrm{C}(8)$ & $1816(2)$ & $3760(4)$ & $937(3)$ & $44(1)$ \\
$\mathrm{C}(9)$ & $397(1)$ & $4108(2)$ & $2635(2)$ & $20(1)$ \\
$\mathrm{N}(1)$ & $-1220(4)$ & $-1774(6)$ & $2331(6)$ & $51(2)$ \\
$\mathrm{C}(10)$ & $-782(11)$ & $-1128(19)$ & $2732(19)$ & $47(5)$ \\
$\mathrm{C}(11)$ & $-230(4)$ & $-511(5)$ & $3249(5)$ & $29(1)$ \\
$\mathrm{C}(12)$ & $485(3)$ & $-486(5)$ & $2883(5)$ & $30(1)$ \\
$\mathrm{C}(13)$ & $673(12)$ & $-1290(20)$ & $1945(18)$ & $43(4)$ \\
$\mathrm{C}(14)$ & $766(4)$ & $-2652(6)$ & $2140(6)$ & $39(2)$ \\
& & & & \\
\hline
\end{tabular}


Table S-8. Intramolecular distances $(\AA)$ and bond angles $\left(^{\circ}\right)$ for complex $7^{\prime}$.

\begin{tabular}{lc}
\hline & \\
$\mathrm{Ni}(1)-\mathrm{Ni}(1) \# 1$ & $0.5234(16)$ \\
$\mathrm{Ni}(1)-\mathrm{C}(12)$ & $1.954(6)$ \\
$\mathrm{Ni}(1)-\mathrm{C}(11)$ & $1.968(6)$ \\
$\mathrm{Ni}(1)-\mathrm{C}(12) \# 1$ & $2.120(6)$ \\
$\mathrm{Ni}(1)-\mathrm{P}(1)$ & $2.1792(18)$ \\
$\mathrm{Ni}(1)-\mathrm{C}(11) \# 1$ & $2.181(6)$ \\
$\mathrm{Ni}(1)-\mathrm{P}(1) \# 1$ & $2.1945(18)$ \\
$\mathrm{P}(1)-\mathrm{C}(9)$ & $1.848(3)$ \\
$\mathrm{P}(1)-\mathrm{C}(5)$ & $1.883(3)$ \\
$\mathrm{P}(1)-\mathrm{C}(1)$ & $1.885(3)$ \\
$\mathrm{P}(1)-\mathrm{Ni}(1) \# 1$ & $2.1944(18)$ \\
$\mathrm{C}(1)-\mathrm{C}(3)$ & $1.519(5)$ \\
$\mathrm{C}(1)-\mathrm{C}(4)$ & $1.520(5)$ \\
$\mathrm{C}(1)-\mathrm{C}(2)$ & $1.532(5)$ \\
$\mathrm{C}(5)-\mathrm{C}(8)$ & $1.526(5)$ \\
$\mathrm{C}(5)-\mathrm{C}(7)$ & $1.526(5)$ \\
$\mathrm{C}(5)-\mathrm{C}(6)$ & $1.532(5)$ \\
$\mathrm{C}(9)-\mathrm{C}(9) \# 1$ & $1.528(5)$ \\
$\mathrm{N}(1)-\mathrm{C}(10)$ & $1.17(3)$ \\
$\mathrm{N}(1)-\mathrm{C}(14) \# 1$ & $1.415(10)$ \\
$\mathrm{N}(1)-\mathrm{C}(13) \# 1$ & $1.45(3)$ \\
$\mathrm{N}(1)-\mathrm{C}(12) \# 1$ & $1.967(9)$ \\
$\mathrm{C}(10)-\mathrm{C}(13) \# 1$ & $0.48(3)$ \\
$\mathrm{C}(10)-\mathrm{C}(12) \# 1$ & $1.19(2)$ \\
$\mathrm{C}(10)-\mathrm{C}(11)$ & $1.38(3)$ \\
$\mathrm{C}(10)-\mathrm{C}(14) \# 1$ & $1.61(2)$ \\
$\mathrm{C}(11)-\mathrm{C}(13) \# 1$ & $1.19(3)$ \\
$\mathrm{C}(11)-\mathrm{C}(12)$ & $1.441(9)$ \\
$\mathrm{C}(11)-\mathrm{C}(12) \# 1$ & $1.498(9)$ \\
$\mathrm{C}(11)-\mathrm{Ni}(1) \# 1$ & $2.181(6)$ \\
$\mathrm{C}(12)-\mathrm{C}(10) \# 1$ & $1.19(2)$ \\
$\mathrm{C}(12)-\mathrm{C}(11) \# 1$ & $1.498(9)$ \\
$\mathrm{C}(12)-\mathrm{C}(13)$ & $1.511(19)$ \\
$\mathrm{C}(12)-\mathrm{N}(1) \# 1$ & $1.966(9)$ \\
$\mathrm{C}(12)-\mathrm{Ni}(1) \# 1$ & $2.119(6)$ \\
$\mathrm{C}(13)-\mathrm{C}(10) \# 1$ & $0.48(3)$ \\
$\mathrm{C}(13)-\mathrm{C}(11) \# 1$ & $1.19(3)$ \\
$\mathrm{C}(13)-\mathrm{N}(1) \# 1$ & $1.45(3)$ \\
$\mathrm{C}(13)-\mathrm{C}(14)$ & $1.46(3)$ \\
$\mathrm{C}(14)-\mathrm{N}(1) \# 1$ & $1.414(10)$ \\
$\mathrm{C}(14)-\mathrm{C}(10) \# 1$ & $1.61(2)$ \\
$\mathrm{Ni}(1) \# 1-\mathrm{Ni}(1)-\mathrm{C}(12)$ & $101.3(3)$ \\
$\mathrm{Ni}(1) \# 1-\mathrm{Ni}(1)-\mathrm{C}(11)$ & $107.2(3)$ \\
$\mathrm{C}(12)-\mathrm{Ni}(1)-\mathrm{C}(11)$ & $43.1(3)$ \\
$\mathrm{Ni}(1) \# 1-\mathrm{Ni}(1)-\mathrm{C}(12) \# 1$ & $64.7(3)$ \\
$\mathrm{C}(12)-\mathrm{Ni}(1)-\mathrm{C}(12) \# 1$ & $60.1(3)$ \\
$\mathrm{C}(11)-\mathrm{Ni}(1)-\mathrm{C}(12) \# 1$ & $42.8(3)$ \\
$\mathrm{Ni}(1) \# 1-\mathrm{Ni}(1)-\mathrm{P}(1)$ & $84.8(3)$ \\
$\mathrm{C}(12)-\mathrm{Ni}(1)-\mathrm{P}(1)$ & $109.5(2)$ \\
& \\
& \\
&
\end{tabular}




$\begin{array}{lc}\text { C(11)-Ni(1)-P(1) } & 151.0(2) \\ \text { C(12)\#1-Ni(1)-P(1) } & 142.6(2) \\ \text { Ni(1)\#1-Ni(1)-C(11)\#1 } & 59.5(2) \\ \text { C(12)-Ni(1)-C(11)\#1 } & 42.0(3) \\ \text { C(11)-Ni(1)-C(11)\#1 } & 60.9(3) \\ \text { C(12)\#1-Ni(1)-C(11)\#1 } & 39.1(2) \\ \text { P(1)-Ni(1)-C(11)\#1 } & 107.33(19) \\ \text { Ni(1)\#1-Ni(1)-P(1)\#1 } & 81.5(3) \\ \text { C(12)-Ni(1)-P(1)\#1 } & 157.9(2) \\ \text { C(11)-Ni(1)-P(1)\#1 } & 115.0(2) \\ \text { C(12)\#1-Ni(1)-P(1)\#1 } & 103.06(19) \\ \text { P(1)-Ni(1)-P(1)\#1 } & 92.49(4) \\ \text { C(11)\#1-Ni(1)-P(1)\#1 } & 133.22(19) \\ \text { C(9)-P(1)-C(5) } & 104.41(14) \\ \text { C(9)-P(1)-C(1) } & 102.29(14) \\ \text { C(5)-P(1)-C(1) } & 109.07(14) \\ \text { C(9)-P(1)-Ni(1) } & 103.77(10) \\ \text { C(5)-P(1)-Ni(1) } & 125.46(11) \\ \text { C(1)-P(1)-Ni(1) } & 109.16(11) \\ \text { C(9)-P(1)-Ni(1)\#1 } & 105.53(10) \\ \text { C(5)-P(1)-Ni(1)\#1 } & 112.45(11) \\ \text { C(1)-P(1)-Ni(1)\#1 } & 121.17(11) \\ \text { Ni(1)-P(1)-Ni(1)\#1 } & 13.74(4) \\ \text { C(3)-C(1)-C(4) } & 109.0(3) \\ \text { C(3)-C(1)-C(2) } & 107.7(3) \\ \text { C(4)-C(1)-C(2) } & 108.6(3) \\ \text { C(3)-C(1)-P(1) } & 110.6(2) \\ \text { C(4)-C(1)-P(1) } & 115.5(2) \\ \text { C(2)-C(1)-P(1) } & 105.1(2) \\ \text { C(8)-C(5)-C(7) } & 107.7(3) \\ \text { C(8)-C(5)-C(6) } & 109.2(3) \\ \text { C(7)-C(5)-C(6) } & 107.4(3) \\ \text { C(8)-C(5)-P(1) } & 115.1(2) \\ \text { C(7)-C(5)-P(1) } & 107.9(2) \\ \text { C(6)-C(5)-P(1) } & 109.4(2) \\ \text { C(9)\#1-C(9)-P(1) } & 112.49(12) \\ \text { C(10)-N(1)-C(14)\#1 } & 76.5(9) \\ \text { C(10)-N(1)-C(13)\#1 } & 17.5(12) \\ \text { C(14)\#1-N(1)-C(13)\#1 } & 61.4(8) \\ \text { C(10)-N(1)-C(12)\#1 } & 33.8(11) \\ \text { C(14)\#1-N(1)-C(12)\#1 } & 95.5(5) \\ \text { C(13)\#1-N(1)-C(12)\#1 } & 49.7(8) \\ \text { C(13)\#1-C(10)-N(1) } & 116(6) \\ \text { C(13)\#1-C(10)-C(12)\#1 } & 124(6) \\ \text { N(1)-C(10)-C(12)\#1 } & 113(2) \\ \text { C(13)\#1-C(10)-C(11) } & 58(5) \\ \text { N(1)-C(10)-C(11) } & 172.7(15) \\ \text { C(12)\#1-C(10)-C(11) } & 71.0(12) \\ \text { C(13)\#1-C(10)-C(14)\#1 } & 64(5) \\ \text { N(1)-C(10)-C(14)\#1 } & 58.5(10) \\ \text { C(12)\#1-C(10)-C(14)\#1 } & 129(2) \\ \text { C(11)-C(10)-C(14)\#1 } & 114.2(11) \\ \text { C(13)\#1-C(11)-C(10) } & 20.1(11) \\ \text { C(13)\#1-C(11)-C(12) } & 127.2(13) \\ \text { C(10)-C(11)-C(12) } & 123.9(12) \\ \text { C(13)\#1-C(11)-C(12)\#1 } & 67.2(10) \\ & \end{array}$




$\begin{array}{lc}\text { C(10)-C(11)-C(12)\#1 } & 48.5(9) \\ \text { C(12)-C(11)-C(12)\#1 } & 88.1(5) \\ \text { C(13)\#1-C(11)-Ni(1) } & 136.8(9) \\ \text { C(10)-C(11)-Ni(1) } & 116.8(7) \\ \text { C(12)-C(11)-Ni(1) } & 67.9(3) \\ \text { C(12)\#1-C(11)-Ni(1) } & 74.0(3) \\ \text { C(13)\#1-C(11)-Ni(1)\#1 } & 125.3(9) \\ \text { C(10)-C(11)-Ni(1)\#1 } & 105.2(7) \\ \text { C(12)-C(11)-Ni(1)\#1 } & 68.1(3) \\ \text { C(12)\#1-C(11)-Ni(1)\#1 } & 60.8(3) \\ \text { Ni(1)-C(11)-Ni(1)\#1 } & 13.25(6) \\ \text { C(10)\#1-C(12)-C(11) } & 132.0(10) \\ \text { C(10)\#1-C(12)-C(11)\#1 } & 60.4(12) \\ \text { C(11)-C(12)-C(11)\#1 } & 91.9(5) \\ \text { C(10)\#1-C(12)-C(13) } & 15.4(17) \\ \text { C(11)-C(12)-C(13) } & 119.3(9) \\ \text { C(11)\#1-C(12)-C(13) } & 46.7(11) \\ \text { C(10)\#1-C(12)-Ni(1) } & 130.4(14) \\ \text { C(11)-C(12)-Ni(1) } & 69.0(3) \\ \text { C(11)\#1-C(12)-Ni(1) } & 77.1(3) \\ \text { C(13)-C(12)-Ni(1) } & 121.5(12) \\ \text { C(10)\#1-C(12)-N(1)\#1 } & 33.4(12) \\ \text { C(11)-C(12)-N(1)\#1 } & 134.8(5) \\ \text { C(11)\#1-C(12)-N(1)\#1 } & 93.6(5) \\ \text { C(13)-C(12)-N(1)\#1 } & 47.1(10) \\ \text { Ni(1)-C(12)-N(1)\#1 } & 155.4(4) \\ \text { C(10)\#1-C(12)-Ni(1)\#1 } & 117.5(14) \\ \text { C(11)-C(12)-Ni(1)\#1 } & 72.7(3) \\ \text { C(11)\#1-C(12)-Ni(1)\#1 } & 63.2(3) \\ \text { C(13)-C(12)-Ni(1)\#1 } & 107.7(11) \\ \text { Ni(1)-C(12)-Ni(1)\#1 } & 14.01(6) \\ \text { N(1)\#1-C(12)-Ni(1)\#1 } & 146.9(4) \\ \text { C(10)\#1-C(13)-C(11)\#1 } & 102(6) \\ \text { C(10)\#1-C(13)-N(1)\#1 } & 47(5) \\ \text { C(11)\#1-C(13)-N(1)\#1 } & 148.4(15) \\ \text { C(10)\#1-C(13)-C(14) } & 99(6) \\ \text { C(11)\#1-C(13)-C(14) } & 142.1(16) \\ \text { N(1)\#1-C(13)-C(14) } & 58.0(12) \\ \text { C(10)\#1-C(13)-C(12) } & 41(5) \\ \text { C(11)\#1-C(13)-C(12) } & 66.1(11) \\ \text { N(1)\#1-C(13)-C(12) } & 83.1(11) \\ \text { C(14)-C(13)-C(12) } & 116.5(18) \\ \text { N(1)\#1-C(14)-C(13) } & 60.5(8) \\ \text { N(1)\#1-C(14)-C(10)\#1 } & 45.0(8) \\ \text { C(13)-C(14)-C(10)\#1 } & 17.2(14) \\ & \end{array}$

Symmetry transformations used to generate equivalent atoms:

$\# 1-\mathrm{x}, \mathrm{y},-\mathrm{z}+1 / 2$ 
Table S-9. U(aniso) values for complex 7'.

\begin{tabular}{lcccccc}
\hline & & & & & \\
& $\mathrm{U} 11$ & $\mathrm{U} 22$ & $\mathrm{U} 33$ & $\mathrm{U} 23$ & $\mathrm{U} 13$ & $\mathrm{U} 12$ \\
\hline $\mathrm{Ni}(1)$ & $15(1)$ & $11(1)$ & $19(1)$ & $1(1)$ & $0(1)$ & $0(1)$ \\
$\mathrm{P}(1)$ & $13(1)$ & $14(1)$ & $22(1)$ & $2(1)$ & $1(1)$ & $1(1)$ \\
$\mathrm{C}(1)$ & $15(1)$ & $46(2)$ & $31(2)$ & $7(2)$ & $-4(1)$ & $5(1)$ \\
$\mathrm{C}(2)$ & $30(2)$ & $146(5)$ & $35(2)$ & $40(3)$ & $-5(2)$ & $15(3)$ \\
$\mathrm{C}(3)$ & $23(2)$ & $43(2)$ & $69(3)$ & $10(2)$ & $-6(2)$ & $11(2)$ \\
$\mathrm{C}(4)$ & $28(2)$ & $59(2)$ & $51(2)$ & $-18(2)$ & $-15(2)$ & $5(2)$ \\
$\mathrm{C}(5)$ & $20(2)$ & $41(2)$ & $26(2)$ & $2(1)$ & $8(1)$ & $2(1)$ \\
$\mathrm{C}(6)$ & $42(2)$ & $67(3)$ & $41(2)$ & $-19(2)$ & $7(2)$ & $12(2)$ \\
$\mathrm{C}(7)$ & $27(2)$ & $66(2)$ & $25(2)$ & $10(2)$ & $6(1)$ & $2(2)$ \\
$\mathrm{C}(8)$ & $27(2)$ & $62(3)$ & $44(2)$ & $21(2)$ & $9(2)$ & $-8(2)$ \\
$\mathrm{C}(9)$ & $19(1)$ & $11(1)$ & $30(2)$ & $-1(1)$ & $3(1)$ & $-3(1)$ \\
$\mathrm{N}(1)$ & $48(4)$ & $42(4)$ & $63(5)$ & $-6(3)$ & $17(3)$ & $-15(3)$ \\
$\mathrm{C}(10)$ & $27(7)$ & $8(5)$ & $109(16)$ & $22(7)$ & $16(8)$ & $0(5)$ \\
$\mathrm{C}(11)$ & $44(4)$ & $13(3)$ & $30(3)$ & $1(2)$ & $9(3)$ & $-6(3)$ \\
$\mathrm{C}(12)$ & $34(3)$ & $10(3)$ & $46(4)$ & $6(3)$ & $-5(3)$ & $1(3)$ \\
$\mathrm{C}(13)$ & $33(7)$ & $19(7)$ & $78(11)$ & $-19(6)$ & $22(7)$ & $-15(4)$ \\
$\mathrm{C}(14)$ & $58(5)$ & $14(3)$ & $45(4)$ & $0(3)$ & $15(3)$ & $7(3)$ \\
& & & & & \\
\hline
\end{tabular}


Table S-10. Hydrogen atom coordinates for complex 7'.

\begin{tabular}{lcccc}
\hline & & & & \\
& $\mathrm{x}$ & $\mathrm{y}$ & $\mathrm{z}$ & $\mathrm{U}(\mathrm{eq})$ \\
\hline $\mathrm{H}(2 \mathrm{~A})$ & 1597 & 2035 & 4904 & 106 \\
$\mathrm{H}(2 \mathrm{~B})$ & 1024 & 1267 & 4248 & 106 \\
$\mathrm{H}(2 \mathrm{C})$ & 885 & 2689 & 4541 & 106 \\
$\mathrm{H}(3 \mathrm{~A})$ & 2488 & 1471 & 3549 & 67 \\
$\mathrm{H}(3 \mathrm{~B})$ & 2297 & 1699 & 2350 & 67 \\
$\mathrm{H}(3 \mathrm{C})$ & 1879 & 686 & 2990 & 67 \\
$\mathrm{H}(4 \mathrm{~A})$ & 2336 & 3623 & 4056 & 69 \\
$\mathrm{H}(4 \mathrm{~B})$ & 1641 & 4378 & 3768 & 69 \\
$\mathrm{H}(4 \mathrm{C})$ & 2171 & 4038 & 2885 & 69 \\
$\mathrm{H}(6 \mathrm{~A})$ & 1592 & 1691 & -186 & 75 \\
$\mathrm{H}(6 \mathrm{~B})$ & 1009 & 1008 & 462 & 75 \\
$\mathrm{H}(6 \mathrm{C})$ & 1774 & 1188 & 956 & 75 \\
$\mathrm{H}(7 \mathrm{~A})$ & 774 & 3508 & -444 & 59 \\
$\mathrm{H}(7 \mathrm{~B})$ & 438 & 4164 & 531 & 59 \\
$\mathrm{H}(7 \mathrm{C})$ & 213 & 2781 & 212 & 59 \\
$\mathrm{H}(8 \mathrm{~A})$ & 1964 & 3844 & 222 & 66 \\
$\mathrm{H}(8 \mathrm{~B})$ & 2201 & 3439 & 1371 & 66 \\
$\mathrm{H}(8 \mathrm{C})$ & 1674 & 4574 & 1196 & 66 \\
$\mathrm{H}(9 \mathrm{~A})$ & 618 & 4777 & 2250 & 24 \\
$\mathrm{H}(9 \mathrm{~B})$ & 470 & 4279 & 3374 & 24 \\
& & & & \\
\hline
\end{tabular}




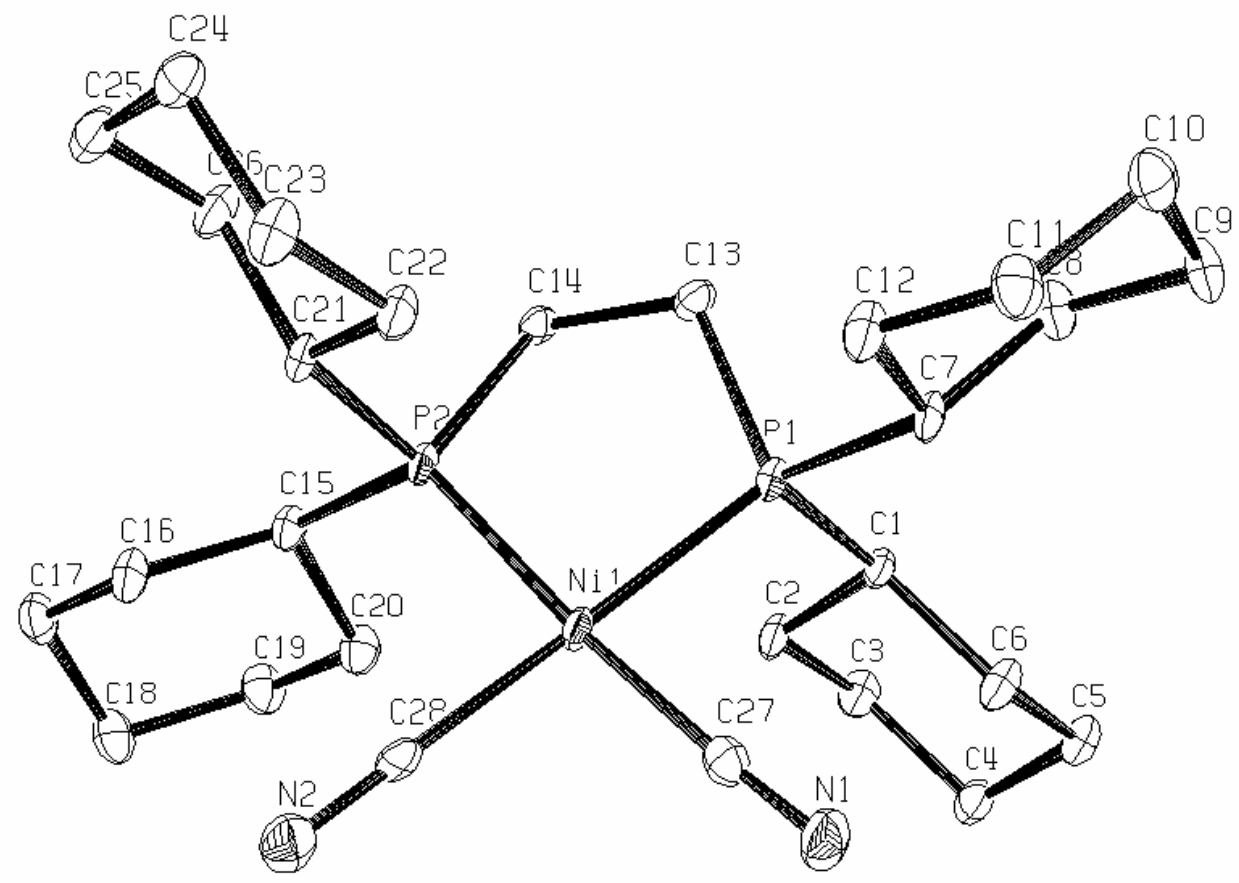

Figure S-4. ORTEP drawing of complex 10 showing 50\% ellipsoids. 
Table S-11. Crystallographic data for complex 10.

\begin{tabular}{|c|c|c|}
\hline Identification code & \multicolumn{2}{|l|}{ JAA25-3 } \\
\hline Empirical formula & \multicolumn{2}{|l|}{$\mathrm{C}_{28} \mathrm{H}_{48} \mathrm{~N}_{2} \mathrm{NiP}_{2}$} \\
\hline Formula weight & \multicolumn{2}{|l|}{533.33} \\
\hline Temperature & \multicolumn{2}{|l|}{$100(2) \mathrm{K}$} \\
\hline Wavelength & \multicolumn{2}{|l|}{$0.71073 \AA$} \\
\hline Crystal system & \multicolumn{2}{|l|}{ Triclinic } \\
\hline Space group & \multicolumn{2}{|l|}{$\mathrm{P} 1$} \\
\hline \multirow[t]{3}{*}{ Unit cell dimensions } & $\mathrm{a}=7.4217(18) \AA$ & $\alpha=93.263(4)^{\circ}$ \\
\hline & $\mathrm{b}=9.326(2) \AA$ & $\beta=108.721(4)^{\circ}$. \\
\hline & $\mathrm{c}=10.718(3) \AA$ & $\gamma=101.016(4)^{\circ}$ \\
\hline Volume & \multicolumn{2}{|l|}{$684.1(3) \AA^{3}$} \\
\hline $\mathrm{Z}$ & \multicolumn{2}{|l|}{1} \\
\hline Density (calculated) & \multicolumn{2}{|l|}{$1.295 \mathrm{Mg} / \mathrm{m}^{3}$} \\
\hline Absorption coefficient & \multicolumn{2}{|l|}{$0.845 \mathrm{~mm}^{-1}$} \\
\hline $\mathrm{F}(000)$ & \multicolumn{2}{|l|}{288} \\
\hline Crystal size & \multicolumn{2}{|c|}{$0.08 \times 0.14 \times 0.17 \mathrm{~mm}^{3}$} \\
\hline Theta range for data collection & \multicolumn{2}{|l|}{2.02 to $28.28^{\circ}$. } \\
\hline Index ranges & \multicolumn{2}{|c|}{$-9<=\mathrm{h}<=9,-12<=\mathrm{k}<=12,-12<=\mathrm{l}<=13$} \\
\hline Reflections collected & \multicolumn{2}{|l|}{4747} \\
\hline Independent reflections & \multicolumn{2}{|c|}{$4180[\mathrm{R}(\mathrm{int})=0.0220]$} \\
\hline Completeness to theta $=28.28^{\circ}$ & \multicolumn{2}{|l|}{$83.1 \%$} \\
\hline Refinement method & \multicolumn{2}{|c|}{ Full-matrix least-squares on $\mathrm{F}^{2}$} \\
\hline Data / restraints / parameters & \multicolumn{2}{|l|}{$4180 / 3 / 298$} \\
\hline Goodness-of-fit on $\mathrm{F}^{2}$ & \multicolumn{2}{|l|}{1.062} \\
\hline Final $R$ indices $[\mathrm{I}>2 \operatorname{sigma}(\mathrm{I})]$ & \multicolumn{2}{|c|}{$\mathrm{R} 1=0.0346, \mathrm{wR} 2=0.0833$} \\
\hline $\mathrm{R}$ indices (all data) & \multicolumn{2}{|c|}{$\mathrm{R} 1=0.0353, \mathrm{wR} 2=0.0837$} \\
\hline Absolute structure parameter & \multicolumn{2}{|l|}{$0.022(12)$} \\
\hline Largest diff. peak and hole & \multicolumn{2}{|c|}{0.847 and -0.548 e. $\AA^{-3}$} \\
\hline
\end{tabular}


Table S-12. Positional parameters and U(eq) for complex 10.

\begin{tabular}{|c|c|c|c|c|}
\hline & $\mathrm{x}$ & $\mathrm{y}$ & z & $\mathrm{U}(\mathrm{eq})$ \\
\hline $\mathrm{Ni}(1)$ & $5455(1)$ & $4380(1)$ & $5725(1)$ & $12(1)$ \\
\hline $\mathrm{P}(1)$ & $3410(1)$ & $5628(1)$ & $4640(1)$ & $12(1)$ \\
\hline $\mathrm{P}(2)$ & $4126(1)$ & $4379(1)$ & $7253(1)$ & $12(1)$ \\
\hline$C(7)$ & $4379(5)$ & $7310(4)$ & $4046(3)$ & $14(1)$ \\
\hline $\mathrm{C}(1)$ & 1541(4) & $4526(3)$ & $3143(3)$ & $12(1)$ \\
\hline $\mathrm{C}(21)$ & $5926(5)$ & $5573(4)$ & $8718(3)$ & $13(1)$ \\
\hline$C(15)$ & $3244(5)$ & 2664(4) & $7855(3)$ & $14(1)$ \\
\hline$C(5)$ & $615(5)$ & $3575(4)$ & $735(4)$ & $18(1)$ \\
\hline $\mathrm{C}(22)$ & $7081(5)$ & 6928(4) & $8363(4)$ & $17(1)$ \\
\hline $\mathrm{C}(23)$ & $8719(5)$ & 7772(4) & 9611(4) & $21(1)$ \\
\hline$C(25)$ & $6749(5)$ & $6827(4)$ & 11018(4) & $22(1)$ \\
\hline$C(4)$ & $-187(5)$ & 2048(4) & $997(4)$ & $18(1)$ \\
\hline $\mathrm{C}(2)$ & $746(5)$ & 2987(4) & $3419(3)$ & $16(1)$ \\
\hline $\mathrm{N}(1)$ & $7469(5)$ & 4841(4) & 3691(3) & $24(1)$ \\
\hline $\mathrm{C}(24)$ & $7909(5)$ & $8165(4)$ & $10686(4)$ & $20(1)$ \\
\hline $\mathrm{C}(8)$ & $2777(5)$ & $8093(4)$ & $3321(4)$ & 19(1) \\
\hline$C(3)$ & $-874(5)$ & 2121(4) & 2189(4) & $17(1)$ \\
\hline $\mathrm{C}(9)$ & $3625(5)$ & $9389(4)$ & 2716(4) & $22(1)$ \\
\hline$C(16)$ & $4776(5)$ & 2196(4) & 9004(4) & $18(1)$ \\
\hline$C(6)$ & $2253(5)$ & $4436(4)$ & 1957(4) & 19(1) \\
\hline$C(17)$ & $3879(5)$ & $795(4)$ & $9445(4)$ & $20(1)$ \\
\hline $\mathrm{C}(11)$ & $6850(5)$ & 9702(4) & $4518(4)$ & $20(1)$ \\
\hline$C(20)$ & $2311(5)$ & $1415(4)$ & 6701(4) & $18(1)$ \\
\hline$C(10)$ & $5265(5)$ & 10461(4) & 3761(4) & $22(1)$ \\
\hline$C(19)$ & $1443(5)$ & $10(4)$ & $7159(4)$ & $21(1)$ \\
\hline$C(28)$ & $7150(5)$ & $3260(4)$ & 6689(4) & $14(1)$ \\
\hline$C(26)$ & $5099(5)$ & 6004(4) & $9786(4)$ & $20(1)$ \\
\hline$C(12)$ & $6024(5)$ & $8393(4)$ & 5121(4) & $18(1)$ \\
\hline$C(18)$ & $2972(5)$ & $-443(4)$ & $8300(4)$ & $22(1)$ \\
\hline$C(27)$ & $6692(5)$ & $4619(4)$ & $4456(4)$ & $16(1)$ \\
\hline $\mathrm{N}(2)$ & $8219(4)$ & 2599(4) & $7268(3)$ & $24(1)$ \\
\hline
\end{tabular}


C(13)

2120(5)

6226(4)

5698(3)

14(1)

C(14)

1941(4)

5149(4)

6687(4)

13(1) 
Table S-13. Intramolecular distances $(\AA)$ and bond angles $\left(^{\circ}\right)$ for complex 10.

\begin{tabular}{|c|c|c|c|}
\hline $\mathrm{Ni}(1)-\mathrm{C}(27)$ & $1.871(4)$ & $C(5)-C(6)$ & $1.524(5)$ \\
\hline $\mathrm{Ni}(1)-\mathrm{C}(28)$ & $1.879(4)$ & $C(22)-C(23)$ & $1.535(5)$ \\
\hline $\mathrm{Ni}(1)-\mathrm{P}(2)$ & $2.1657(11)$ & $C(23)-C(24)$ & $1.518(5)$ \\
\hline $\mathrm{Ni}(1)-\mathrm{P}(1)$ & $2.1693(10)$ & $C(25)-C(24)$ & $1.504(5)$ \\
\hline$P(1)-C(13)$ & $1.827(3)$ & $C(25)-C(26)$ & $1.523(5)$ \\
\hline$P(1)-C(7)$ & $1.834(4)$ & $C(4)-C(3)$ & $1.523(5)$ \\
\hline $\mathrm{P}(1)-\mathrm{C}(1)$ & $1.842(3)$ & $\mathrm{C}(2)-\mathrm{C}(3)$ & $1.525(4)$ \\
\hline $\mathrm{P}(2)-\mathrm{C}(14)$ & $1.837(3)$ & $\mathrm{N}(1)-\mathrm{C}(27)$ & $1.151(5)$ \\
\hline$P(2)-C(21)$ & $1.838(3)$ & $\mathrm{C}(8)-\mathrm{C}(9)$ & $1.529(5)$ \\
\hline$P(2)-C(15)$ & $1.842(4)$ & $\mathrm{C}(9)-\mathrm{C}(10)$ & $1.506(5)$ \\
\hline$C(7)-C(12)$ & $1.527(4)$ & $C(16)-C(17)$ & $1.522(5)$ \\
\hline$C(7)-C(8)$ & $1.534(4)$ & $\mathrm{C}(17)-\mathrm{C}(18)$ & $1.518(5)$ \\
\hline $\mathrm{C}(1)-\mathrm{C}(2)$ & $1.527(5)$ & $\mathrm{C}(11)-\mathrm{C}(10)$ & $1.521(5)$ \\
\hline$C(1)-C(6)$ & $1.529(5)$ & $\mathrm{C}(11)-\mathrm{C}(12)$ & $1.530(5)$ \\
\hline$C(21)-C(22)$ & $1.525(5)$ & $C(20)-C(19)$ & $1.525(5)$ \\
\hline$C(21)-C(26)$ & $1.530(5)$ & $\mathrm{C}(19)-\mathrm{C}(18)$ & $1.521(5)$ \\
\hline$C(15)-C(20)$ & $1.531(5)$ & $\mathrm{C}(28)-\mathrm{N}(2)$ & $1.144(4)$ \\
\hline$C(15)-C(16)$ & $1.533(4)$ & $C(13)-C(14)$ & $1.520(5)$ \\
\hline$C(5)-C(4)$ & $1.513(5)$ & & \\
\hline $\mathrm{C}(27)-\mathrm{Ni}(1)-\mathrm{C}(28)$ & $93.30(16)$ & $\mathrm{C}(14)-\mathrm{P}(2)-\mathrm{C}(15)$ & $102.92(16)$ \\
\hline $\mathrm{C}(27)-\mathrm{Ni}(1)-\mathrm{P}(2)$ & $173.37(12)$ & $\mathrm{C}(21)-\mathrm{P}(2)-\mathrm{C}(15)$ & 106.11(16) \\
\hline $\mathrm{C}(28)-\mathrm{Ni}(1)-\mathrm{P}(2)$ & $89.72(12)$ & $\mathrm{C}(14)-\mathrm{P}(2)-\mathrm{Ni}(1)$ & $109.60(13)$ \\
\hline $\mathrm{C}(27)-\mathrm{Ni}(1)-\mathrm{P}(1)$ & $88.72(11)$ & $\mathrm{C}(21)-\mathrm{P}(2)-\mathrm{Ni}(1)$ & $106.86(12)$ \\
\hline $\mathrm{C}(28)-\mathrm{Ni}(1)-\mathrm{P}(1)$ & $177.71(13)$ & $\mathrm{C}(15)-\mathrm{P}(2)-\mathrm{Ni}(1)$ & $121.78(11)$ \\
\hline $\mathrm{P}(2)-\mathrm{Ni}(1)-\mathrm{P}(1)$ & $88.38(4)$ & $C(12)-C(7)-C(8)$ & $110.2(3)$ \\
\hline $\mathrm{C}(13)-\mathrm{P}(1)-\mathrm{C}(7)$ & $104.95(17)$ & $\mathrm{C}(12)-\mathrm{C}(7)-\mathrm{P}(1)$ & $114.0(2)$ \\
\hline $\mathrm{C}(13)-\mathrm{P}(1)-\mathrm{C}(1)$ & $106.87(15)$ & $C(8)-C(7)-P(1)$ & $112.4(2)$ \\
\hline$C(7)-P(1)-C(1)$ & $104.35(16)$ & $\mathrm{C}(2)-\mathrm{C}(1)-\mathrm{C}(6)$ & $110.7(3)$ \\
\hline $\mathrm{C}(13)-\mathrm{P}(1)-\mathrm{Ni}(1)$ & $109.37(12)$ & $C(2)-C(1)-P(1)$ & $111.6(2)$ \\
\hline $\mathrm{C}(7)-\mathrm{P}(1)-\mathrm{Ni}(1)$ & $118.10(11)$ & $\mathrm{C}(6)-\mathrm{C}(1)-\mathrm{P}(1)$ & $113.0(2)$ \\
\hline $\mathrm{C}(1)-\mathrm{P}(1)-\mathrm{Ni}(1)$ & $112.42(10)$ & $C(22)-C(21)-C(26)$ & $111.0(3)$ \\
\hline $\mathrm{C}(14)-\mathrm{P}(2)-\mathrm{C}(21)$ & $109.09(15)$ & $\mathrm{C}(22)-\mathrm{C}(21)-\mathrm{P}(2)$ & $112.9(2)$ \\
\hline
\end{tabular}




$\begin{array}{ll}\mathrm{C}(26)-\mathrm{C}(21)-\mathrm{P}(2) & 114.4(2) \\ \mathrm{C}(20)-\mathrm{C}(15)-\mathrm{C}(16) & 110.8(3) \\ \mathrm{C}(20)-\mathrm{C}(15)-\mathrm{P}(2) & 110.2(3) \\ \mathrm{C}(16)-\mathrm{C}(15)-\mathrm{P}(2) & 115.0(2) \\ \mathrm{C}(4)-\mathrm{C}(5)-\mathrm{C}(6) & 111.4(3) \\ \mathrm{C}(21)-\mathrm{C}(22)-\mathrm{C}(23) & 110.2(3) \\ \mathrm{C}(24)-\mathrm{C}(23)-\mathrm{C}(22) & 111.0(3) \\ \mathrm{C}(24)-\mathrm{C}(25)-\mathrm{C}(26) & 111.0(3) \\ \mathrm{C}(5)-\mathrm{C}(4)-\mathrm{C}(3) & 111.0(3) \\ \mathrm{C}(3)-\mathrm{C}(2)-\mathrm{C}(1) & 110.8(3) \\ \mathrm{C}(25)-\mathrm{C}(24)-\mathrm{C}(23) & 111.8(3) \\ \mathrm{C}(9)-\mathrm{C}(8)-\mathrm{C}(7) & 110.3(3) \\ \mathrm{C}(4)-\mathrm{C}(3)-\mathrm{C}(2) & 111.4(3) \\ \mathrm{C}(10)-\mathrm{C}(9)-\mathrm{C}(8) & 111.2(3) \\ \mathrm{C}(17)-\mathrm{C}(16)-\mathrm{C}(15) & 110.5(3) \\ \mathrm{C}(5)-\mathrm{C}(6)-\mathrm{C}(1) & 110.4(3) \\ \mathrm{C}(18)-\mathrm{C}(17)-\mathrm{C}(16) & 111.4(3) \\ \mathrm{C}(10)-\mathrm{C}(11)-\mathrm{C}(12) & 111.6(3) \\ \mathrm{C}(19)-\mathrm{C}(20)-\mathrm{C}(15) & 111.1(3) \\ \mathrm{C}(9)-\mathrm{C}(10)-\mathrm{C}(11) & 111.3(3) \\ \mathrm{C}(18)-\mathrm{C}(19)-\mathrm{C}(20) & 111.1(3) \\ \mathrm{N}(2)-\mathrm{C}(28)-\mathrm{Ni}(1) & 178.3(4) \\ \mathrm{C}(25)-\mathrm{C}(26)-\mathrm{C}(21) & 109.9(3) \\ \mathrm{C}(7)-\mathrm{C}(12)-\mathrm{C}(11) & 110.5(3) \\ \mathrm{C}(17)-\mathrm{C}(18)-\mathrm{C}(19) & 110.8(3) \\ \mathrm{N}(1)-\mathrm{C}(27)-\mathrm{Ni}(1) & 176.5(4) \\ \mathrm{C}(14)-\mathrm{C}(13)-\mathrm{P}(1) & 111.2(2) \\ \mathrm{C}(13)-\mathrm{C}(14)-\mathrm{P}(2) & 110.8(2 \\ & \end{array}$


Table S-14. U(aniso) values for complex 10.

\begin{tabular}{|c|c|c|c|c|c|c|}
\hline & $\mathrm{U}^{11}$ & $\mathrm{U}^{22}$ & $\mathrm{U}^{33}$ & $\mathrm{U}^{23}$ & $\mathrm{U}^{13}$ & $\mathrm{U}^{12}$ \\
\hline $\mathrm{Ni}(1)$ & $14(1)$ & $16(1)$ & $7(1)$ & $4(1)$ & $5(1)$ & $4(1)$ \\
\hline $\mathrm{P}(1)$ & $13(1)$ & $14(1)$ & $8(1)$ & $2(1)$ & $4(1)$ & $2(1)$ \\
\hline $\mathrm{P}(2)$ & $15(1)$ & $15(1)$ & $7(1)$ & $3(1)$ & $5(1)$ & $3(1)$ \\
\hline$C(7)$ & $18(2)$ & $16(2)$ & $8(2)$ & $5(1)$ & $5(1)$ & $1(1)$ \\
\hline $\mathrm{C}(1)$ & $16(1)$ & $12(2)$ & $8(2)$ & $1(1)$ & $4(1)$ & $2(1)$ \\
\hline$C(21)$ & $15(2)$ & $18(2)$ & $7(2)$ & $3(1)$ & $3(1)$ & $3(1)$ \\
\hline$C(15)$ & $17(2)$ & $16(2)$ & $11(2)$ & $4(1)$ & $5(1)$ & $2(1)$ \\
\hline$C(5)$ & $20(2)$ & $22(2)$ & $10(2)$ & $2(2)$ & $5(1)$ & $3(1)$ \\
\hline$C(22)$ & $22(2)$ & $17(2)$ & $10(2)$ & $2(1)$ & $5(1)$ & $1(1)$ \\
\hline$C(23)$ & $20(2)$ & $25(2)$ & $14(2)$ & $3(2)$ & $5(2)$ & $0(1)$ \\
\hline$C(25)$ & $30(2)$ & $24(2)$ & $12(2)$ & $0(2)$ & $6(2)$ & $5(2)$ \\
\hline$C(4)$ & $22(2)$ & $20(2)$ & $11(2)$ & $1(2)$ & $5(1)$ & $4(1)$ \\
\hline$C(2)$ & $21(2)$ & $18(2)$ & $8(2)$ & $3(1)$ & $5(1)$ & $3(1)$ \\
\hline $\mathrm{N}(1)$ & $23(2)$ & $37(2)$ & $20(2)$ & $12(2)$ & $13(2)$ & $12(1)$ \\
\hline$C(24)$ & $23(2)$ & $20(2)$ & $13(2)$ & $-1(2)$ & $2(2)$ & $4(1)$ \\
\hline $\mathrm{C}(8)$ & $19(2)$ & $19(2)$ & $18(2)$ & $8(2)$ & $4(1)$ & $2(1)$ \\
\hline$C(3)$ & $20(2)$ & $14(2)$ & $15(2)$ & $1(1)$ & $6(1)$ & $-2(1)$ \\
\hline $\mathrm{C}(9)$ & $28(2)$ & $22(2)$ & $19(2)$ & $13(2)$ & $7(2)$ & $8(2)$ \\
\hline$C(16)$ & $22(2)$ & $21(2)$ & 11(2) & $6(2)$ & $5(1)$ & $3(1)$ \\
\hline$C(6)$ & $22(2)$ & $22(2)$ & $11(2)$ & $2(2)$ & $8(1)$ & $2(1)$ \\
\hline$C(17)$ & $23(2)$ & $24(2)$ & $17(2)$ & $11(2)$ & $9(2)$ & $9(2)$ \\
\hline $\mathrm{C}(11)$ & $22(2)$ & $17(2)$ & $19(2)$ & $5(2)$ & $6(2)$ & $-1(1)$ \\
\hline$C(20)$ & $20(2)$ & $17(2)$ & $15(2)$ & $3(1)$ & $5(1)$ & $1(1)$ \\
\hline$C(10)$ & $29(2)$ & $18(2)$ & $22(2)$ & $8(2)$ & $12(2)$ & $3(1)$ \\
\hline$C(19)$ & $23(2)$ & $16(2)$ & $20(2)$ & $4(2)$ & $4(2)$ & $2(1)$ \\
\hline$C(28)$ & $11(2)$ & $21(2)$ & $13(2)$ & $1(2)$ & $8(1)$ & $3(1)$ \\
\hline$C(26)$ & $26(2)$ & $22(2)$ & $11(2)$ & $2(2)$ & $9(2)$ & $2(1)$ \\
\hline$C(12)$ & $21(2)$ & $20(2)$ & 11(2) & $3(1)$ & $4(1)$ & $0(1)$ \\
\hline$C(18)$ & $27(2)$ & $19(2)$ & $22(2)$ & $11(2)$ & $9(2)$ & $6(2)$ \\
\hline$C(27)$ & $14(2)$ & $17(2)$ & $15(2)$ & $3(2)$ & $3(2)$ & $4(1)$ \\
\hline
\end{tabular}




\begin{tabular}{lllllll}
$\mathrm{N}(2)$ & $21(2)$ & $32(2)$ & $23(2)$ & $7(2)$ & $12(1)$ & $8(1)$ \\
$\mathrm{C}(13)$ & $13(2)$ & $19(2)$ & $11(2)$ & $1(1)$ & $5(1)$ & $5(1)$ \\
$\mathrm{C}(14)$ & $12(2)$ & $17(2)$ & $12(2)$ & $2(1)$ & $6(1)$ & $4(1)$ \\
\hline
\end{tabular}


Table S-15. Hydrogen atom coordinates for complex 10.

\begin{tabular}{|c|c|c|c|c|}
\hline & $\mathrm{x}$ & $\mathrm{y}$ & z & $\mathrm{U}(\mathrm{eq})$ \\
\hline $\mathrm{H}(7)$ & 4938 & 7002 & 3389 & 17 \\
\hline $\mathrm{H}(1)$ & 450 & 5023 & 2894 & 15 \\
\hline $\mathrm{H}(21)$ & 6875 & 4991 & 9129 & 16 \\
\hline $\mathrm{H}(15)$ & 2207 & 2835 & 8186 & 17 \\
\hline $\mathrm{H}(5 \mathrm{~A})$ & -426 & 4104 & 481 & 21 \\
\hline $\mathrm{H}(5 \mathrm{~B})$ & 1104 & 3496 & 2 & 21 \\
\hline $\mathrm{H}(22 \mathrm{~A})$ & 6217 & 7565 & 7973 & 20 \\
\hline $\mathrm{H}(22 \mathrm{~B})$ & 7638 & 6626 & 7714 & 20 \\
\hline $\mathrm{H}(23 \mathrm{~A})$ & 9660 & 7172 & 9942 & 25 \\
\hline $\mathrm{H}(23 \mathrm{~B})$ & 9386 & 8666 & 9384 & 25 \\
\hline $\mathrm{H}(25 \mathrm{~A})$ & 6210 & 7125 & 11679 & 27 \\
\hline $\mathrm{H}(25 \mathrm{~B})$ & 7602 & 6177 & 11393 & 27 \\
\hline $\mathrm{H}(4 \mathrm{~A})$ & 818 & 1483 & 1162 & 22 \\
\hline $\mathrm{H}(4 \mathrm{~B})$ & -1270 & 1549 & 219 & 22 \\
\hline $\mathrm{H}(2 \mathrm{~A})$ & 1794 & 2465 & 3684 & 19 \\
\hline $\mathrm{H}(2 \mathrm{~B})$ & 242 & 3067 & 4144 & 19 \\
\hline $\mathrm{H}(24 \mathrm{~A})$ & 8979 & 8645 & 11479 & 25 \\
\hline $\mathrm{H}(24 \mathrm{~B})$ & 7082 & 8855 & 10389 & 25 \\
\hline $\mathrm{H}(8 \mathrm{~A})$ & 1756 & 7403 & 2625 & 23 \\
\hline $\mathrm{H}(8 \mathrm{~B})$ & 2205 & 8447 & 3942 & 23 \\
\hline $\mathrm{H}(3 \mathrm{~A})$ & -1301 & 1130 & 2370 & 21 \\
\hline $\mathrm{H}(3 \mathrm{~B})$ & -1980 & 2587 & 1983 & 21 \\
\hline $\mathrm{H}(9 \mathrm{~A})$ & 4105 & 9024 & 2046 & 27 \\
\hline $\mathrm{H}(9 \mathrm{~B})$ & 2606 & 9892 & 2285 & 27 \\
\hline $\mathrm{H}(16 \mathrm{~A})$ & 5845 & 2030 & 8722 & 22 \\
\hline $\mathrm{H}(16 \mathrm{~B})$ & 5294 & 2978 & 9745 & 22 \\
\hline $\mathrm{H}(6 \mathrm{~A})$ & 2699 & 5422 & 1771 & 22 \\
\hline $\mathrm{H}(6 \mathrm{~B})$ & 3344 & 3955 & 2168 & 22 \\
\hline $\mathrm{H}(17 \mathrm{~A})$ & 4879 & 494 & 10148 & 24 \\
\hline $\mathrm{H}(17 \mathrm{~B})$ & 2887 & 988 & 9799 & 24 \\
\hline
\end{tabular}




\begin{tabular}{lrrrr}
$\mathrm{H}(11 \mathrm{~A})$ & 7839 & 10404 & 5221 & 24 \\
$\mathrm{H}(11 \mathrm{~B})$ & 7466 & 9361 & 3921 & 24 \\
$\mathrm{H}(20 \mathrm{~A})$ & 1292 & 1711 & 6009 & 21 \\
$\mathrm{H}(20 \mathrm{~B})$ & 3288 & 1225 & 6330 & 21 \\
$\mathrm{H}(10 \mathrm{~A})$ & 4756 & 10905 & 4378 & 27 \\
$\mathrm{H}(10 \mathrm{~B})$ & 5822 & 11240 & 3343 & 27 \\
$\mathrm{H}(19 \mathrm{~A})$ & 373 & 170 & 7443 & 25 \\
$\mathrm{H}(19 \mathrm{~B})$ & 929 & -777 & 6423 & 25 \\
$\mathrm{H}(26 \mathrm{~A})$ & 4188 & 6625 & 9447 & 23 \\
$\mathrm{H}(26 \mathrm{~B})$ & 4401 & 5126 & 10014 & 23 \\
$\mathrm{H}(12 \mathrm{~A})$ & 7053 & 7897 & 5549 & 22 \\
$\mathrm{H}(12 \mathrm{~B})$ & 5532 & 8743 & 5790 & 22 \\
$\mathrm{H}(18 \mathrm{~A})$ & 2371 & -1309 & 8603 & 26 \\
$\mathrm{H}(18 \mathrm{~B})$ & 3981 & -695 & 7993 & 26 \\
$\mathrm{H}(13 \mathrm{~A})$ & 2820 & 7192 & 6175 & 17 \\
$\mathrm{H}(13 \mathrm{~B})$ & 829 & 6305 & 5149 & 17 \\
$\mathrm{H}(14 \mathrm{~A})$ & 802 & 4357 & 6274 & 15 \\
$\mathrm{H}(14 \mathrm{~B})$ & 1769 & 5650 & 7445 & 15 \\
\hline
\end{tabular}



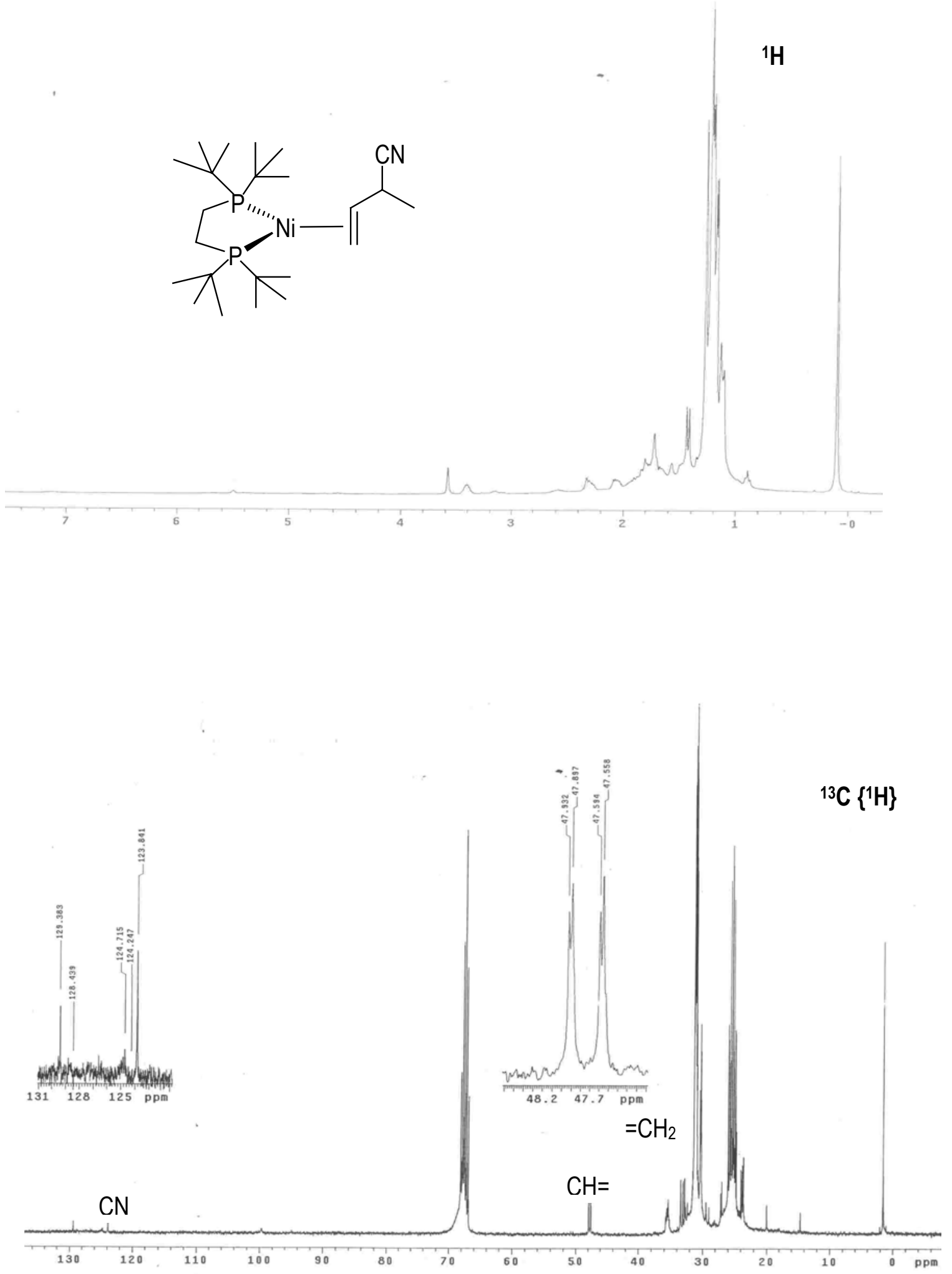

Figure S-5. NMR spectra for 1' 

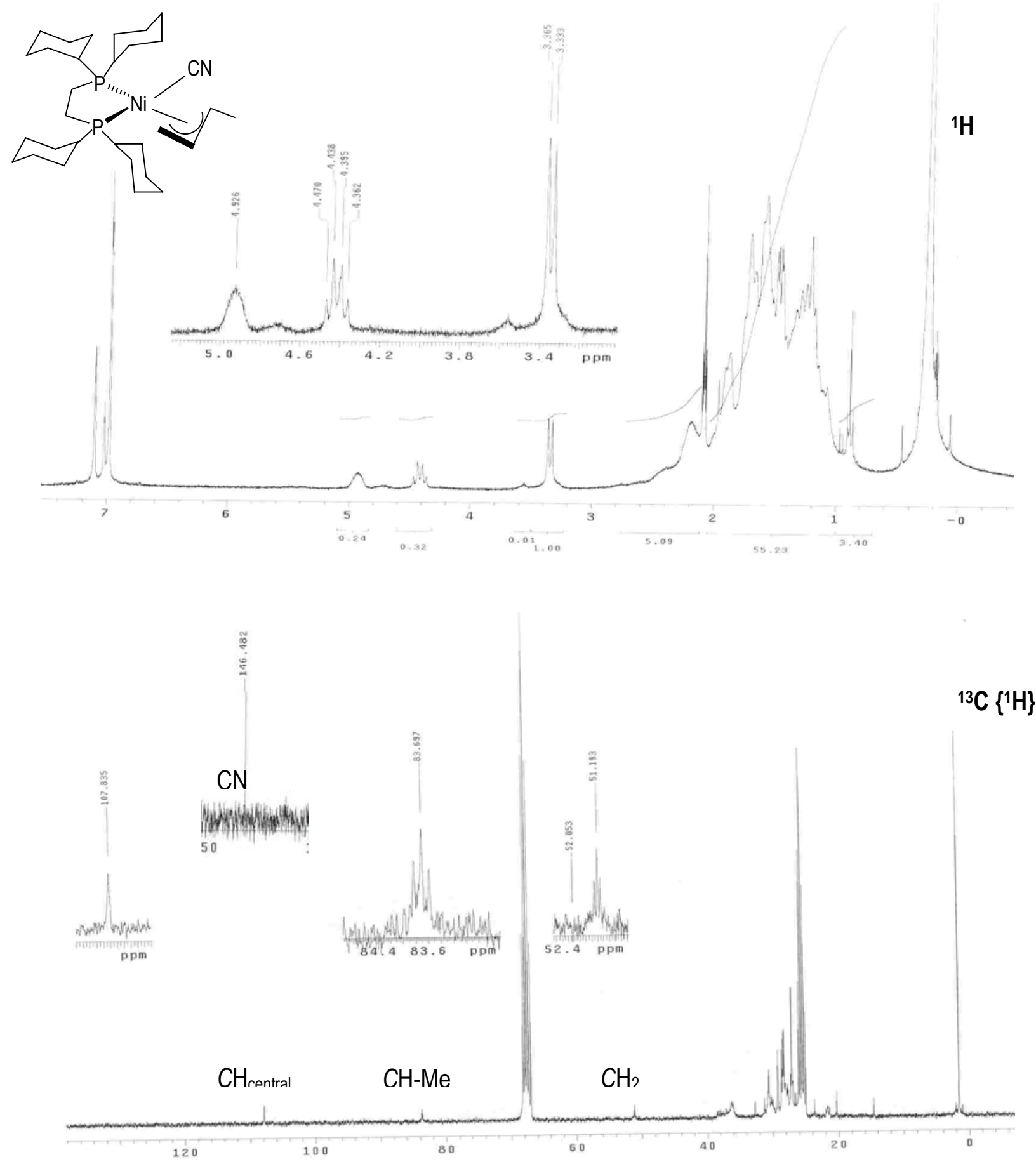

Figure S-6. NMR spectra for 2 

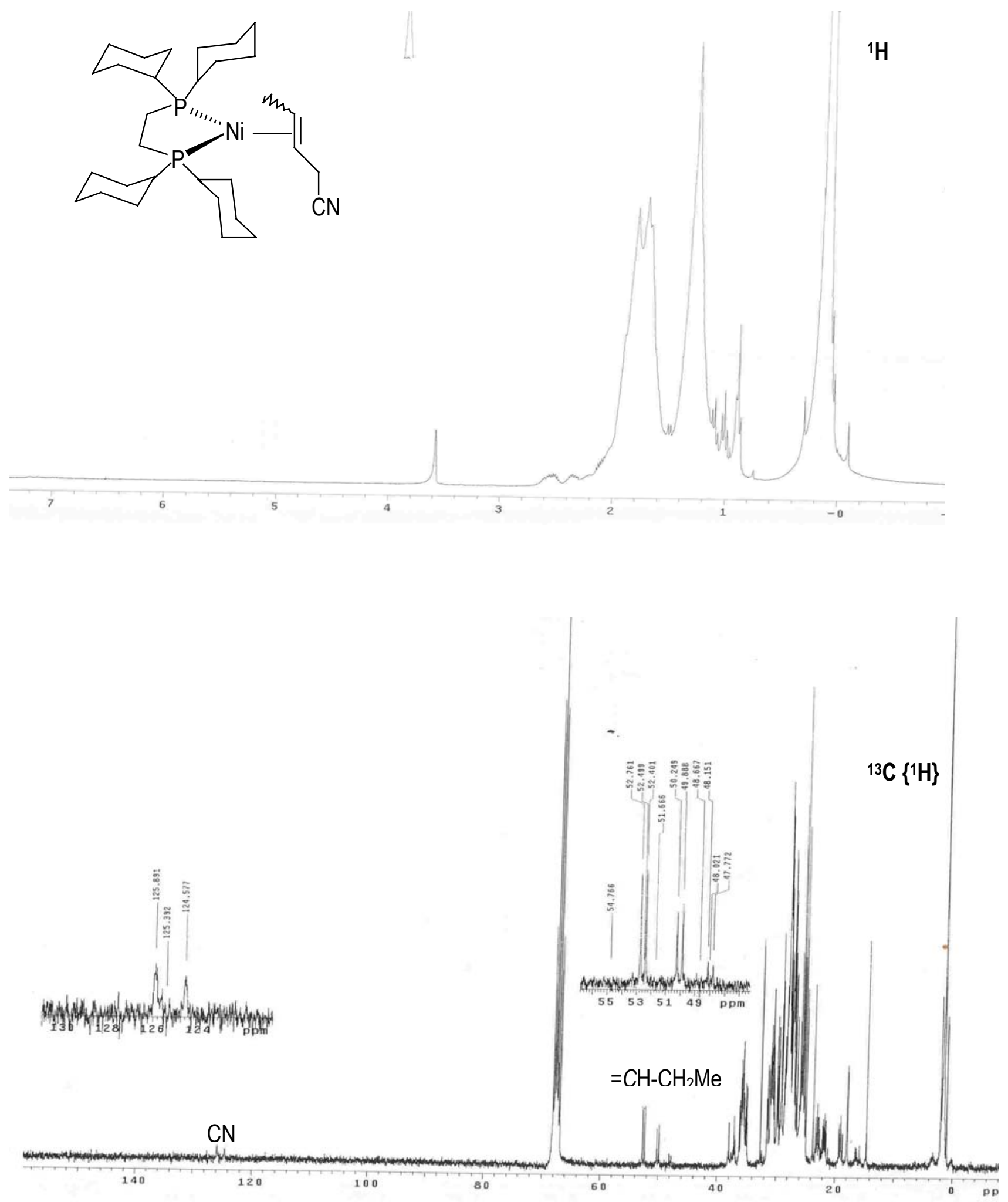

Figure S-7. NMR spectra for 3 and 4 


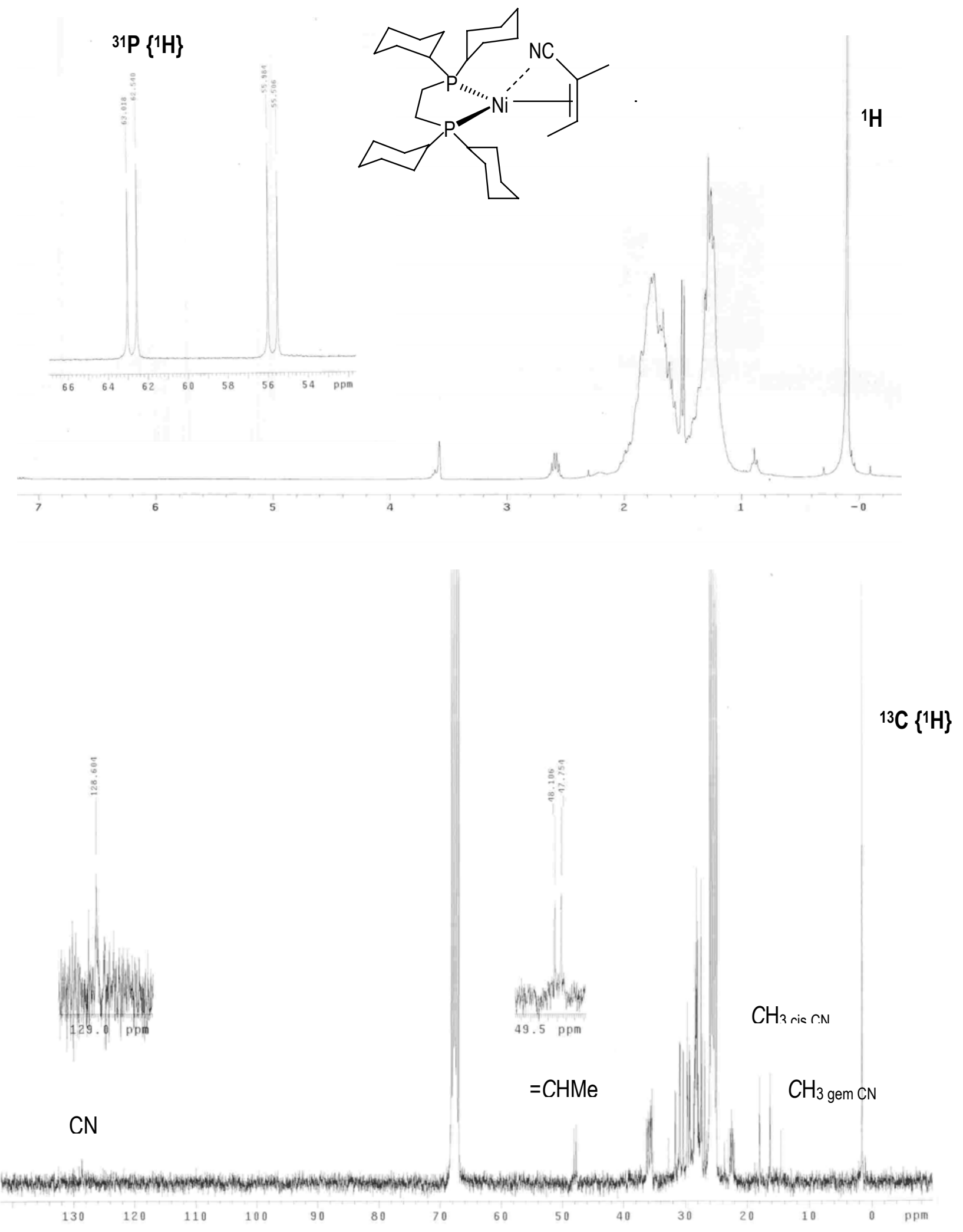

Figure S-8. NMR spectra for $\mathbf{5}$ 

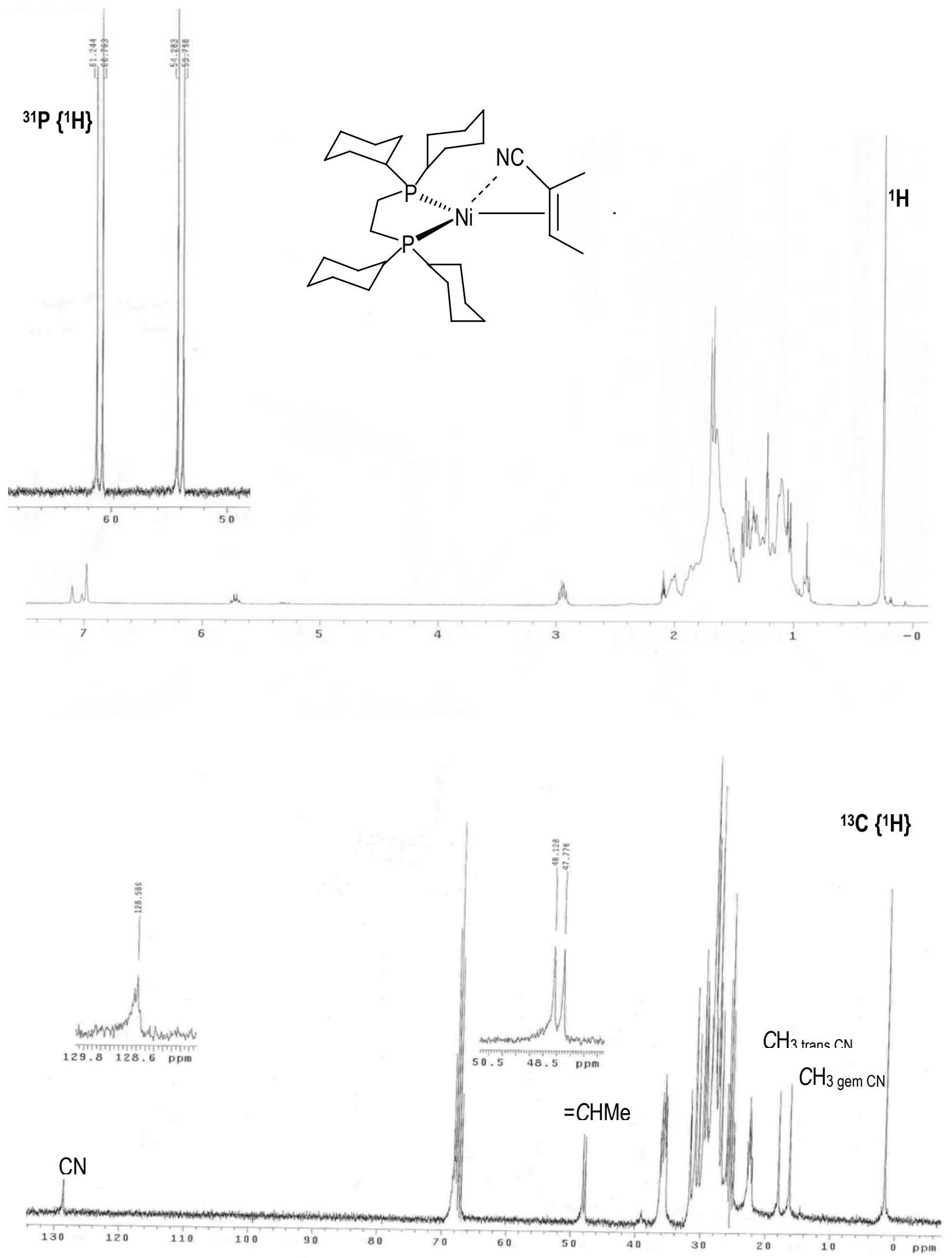

Figure S-9. NMR spectra for 6 

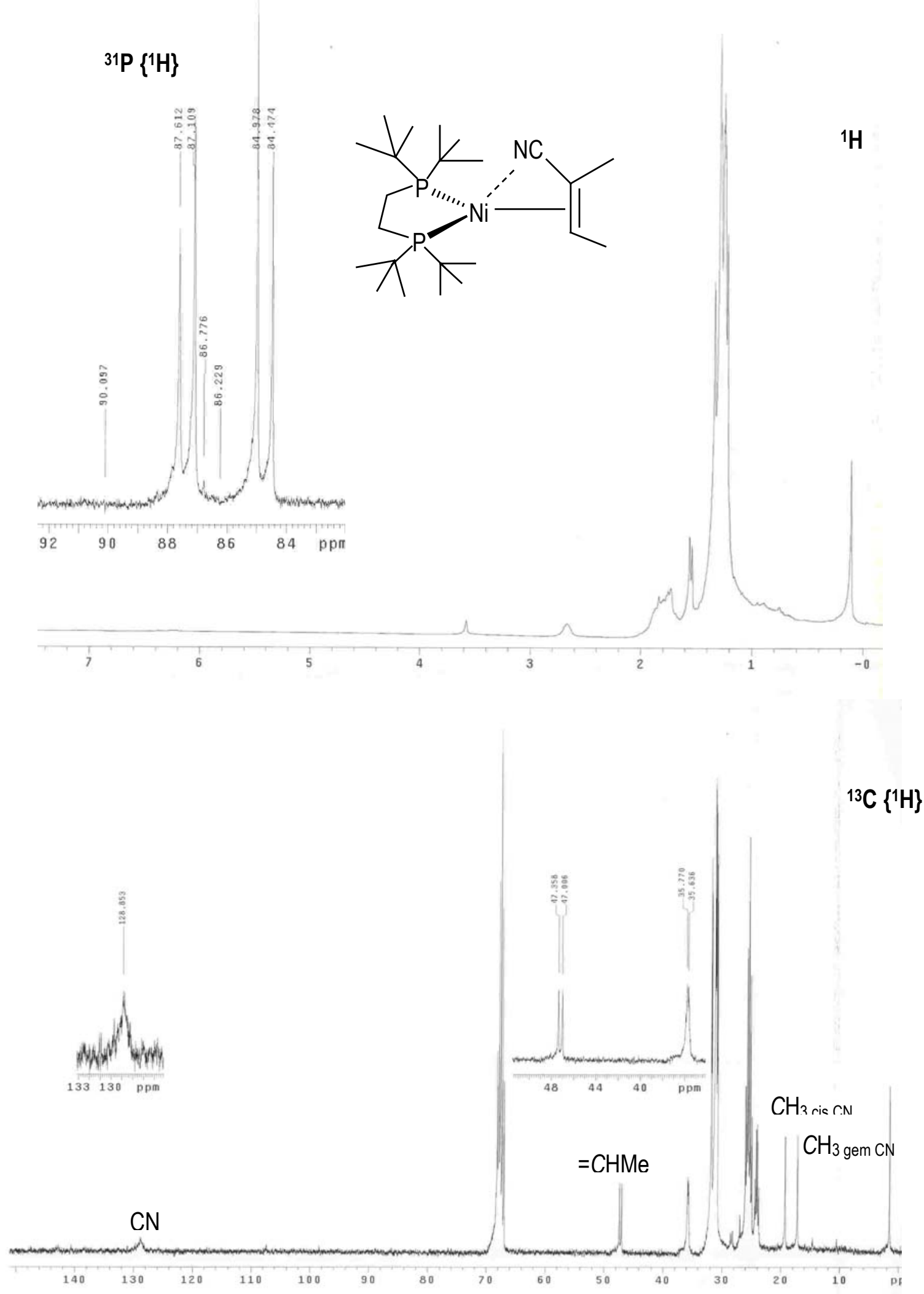

Figure S-10. NMR spectra for 6' 


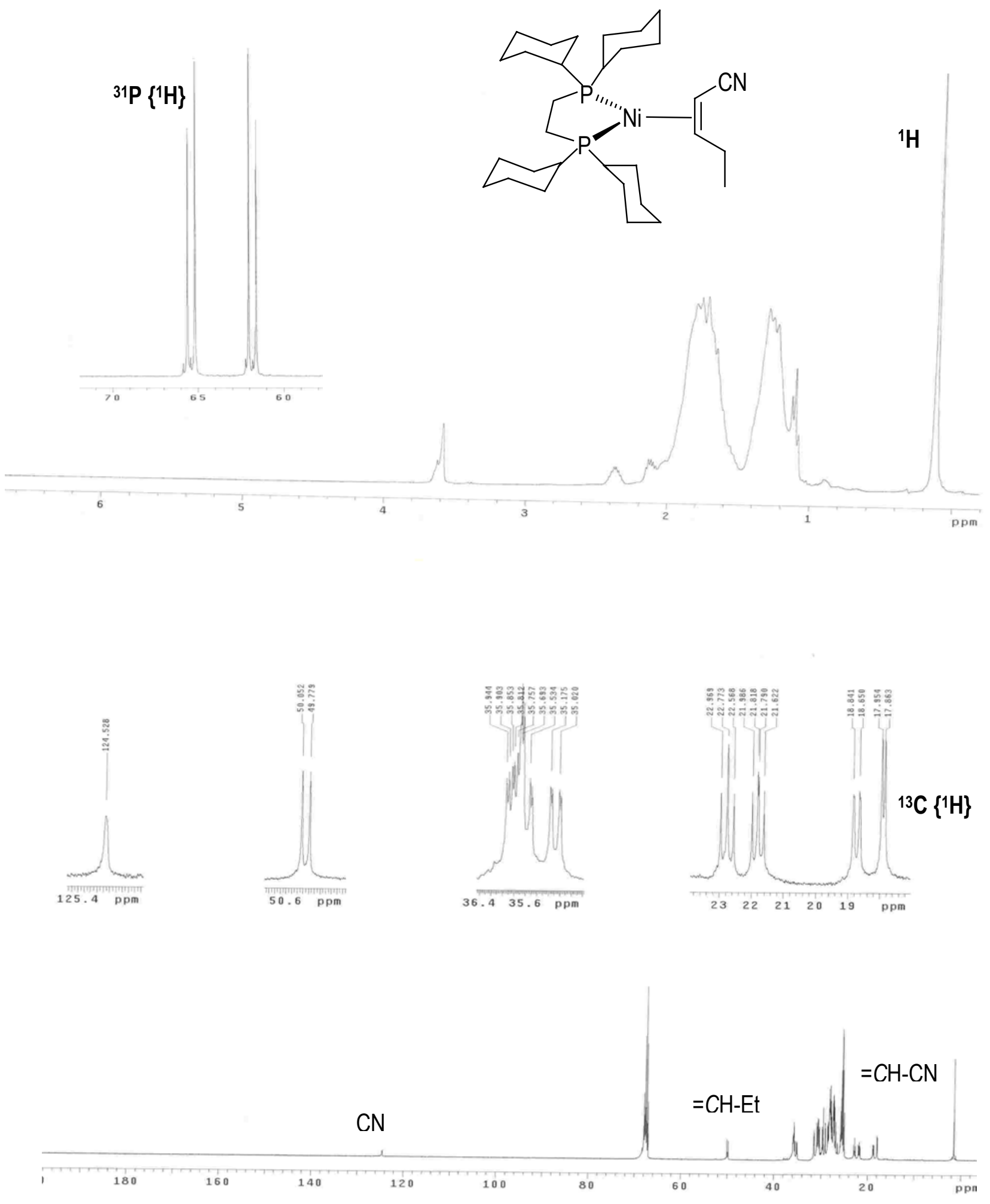

Figure S-11. NMR spectra for 7 

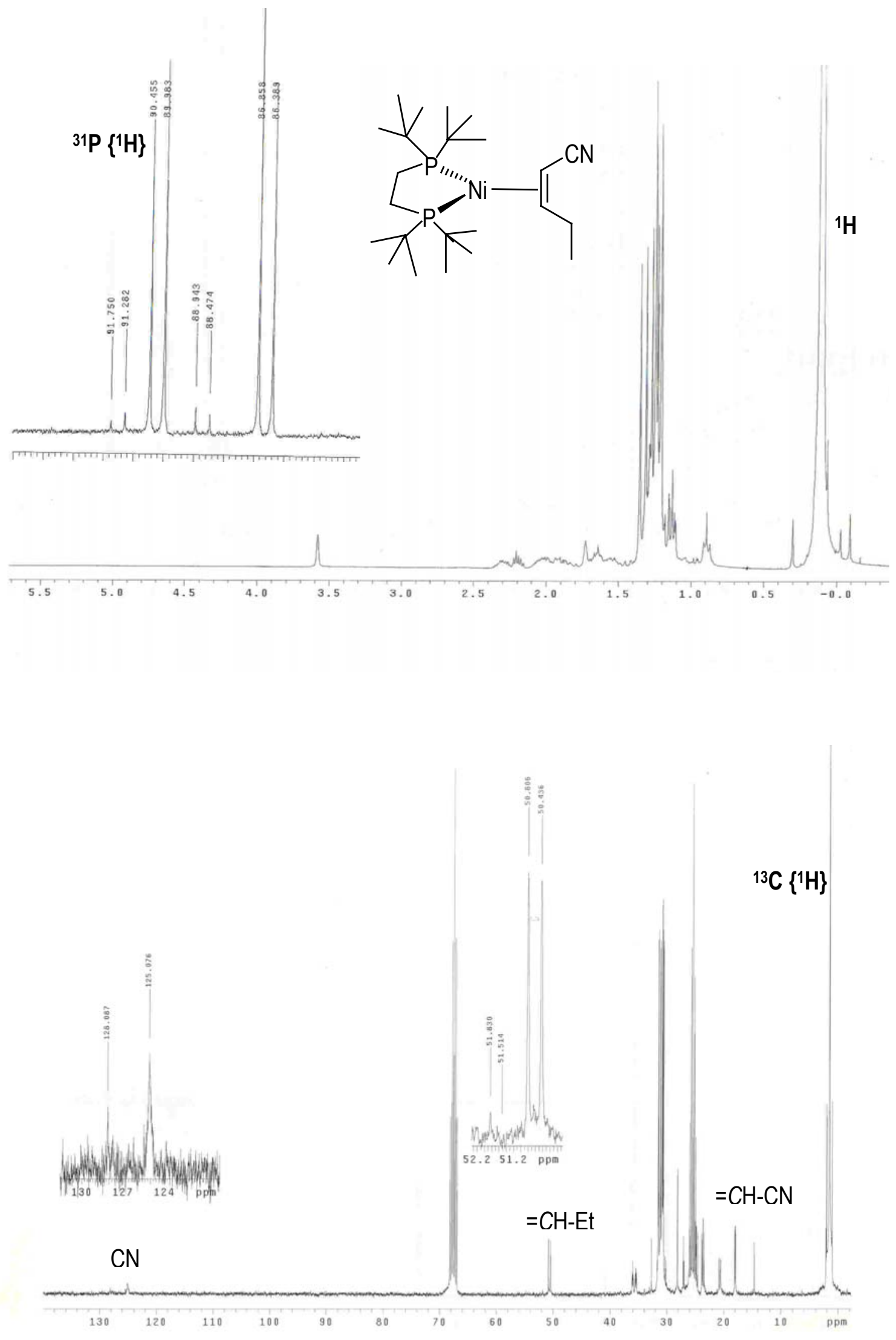

Figure S-12. NMZR spectra for 7' 